Bull. Soc. math. France

133 (3), 2005, p. 363-394

\title{
DISTRIBUTION DES PRÉIMAGES ET DES POINTS PÉRIODIQUES D'UNE CORRESPONDANCE POLYNOMIALE
}

\author{
PAR TIEN-CuOng Dinh
}

À Madame Lê Hông Sâm

RÉSUMÉ. - Nous construisons pour toute correspondance polynomiale $F$ d'exposant de Lojasiewicz $\ell>1$ une mesure d'équilibre $\mu$. Nous montrons que $\mu$ est approximable par les préimages d'un point générique et que les points périodiques répulsifs sont équidistribués sur le support de $\mu$. En utilisant ces résultats, nous donnons une caractérisation des ensembles d'unicité pour les polynômes.

ABStRact (Distribution of preimages and periodic points of a polynomial correspondence)

We construct an equilibrium measure $\mu$ for a polynomial correspondence $F$ of Lojasiewicz exponent $\ell>1$. We then show that $\mu$ can be built as the distribution of preimages of a generic point and that the repelling periodic points are equidistributed on the support of $\mu$. Using these results, we will give a characterization of infinite uniqueness sets for polynomials.

\section{Introduction}

Un compact $K$ de $\mathbb{C}$ est un ensemble d'unicité si pour tout couple de polynômes non constants $f$ et $g$, la relation $f^{-1}(K)=g^{-1}(K)$ implique $f=g$. Ostrovskii, Pakovitch et Zaidenberg [29] ont montré que si $f$ et $g$ sont deux

Texte reçu le 25 mai 2003, accepté le 3 février 2004

Tien-Cuong Dinh, Université Paris-Sud, Mathématique, Bât. 425, 91405 Orsay (France)

E-mail : TienCuong.Dinh@math.u-psud.fr

Classification mathématique par sujets (2000). - 37F, 32H, 32H30, 32H50.

Mots clefs. - Correspondance, mesure d'équilibre, ensemble exceptionnel, point périodique, ensemble d'unicité. 
polynômes de même degré vérifiant $f^{-1}(K)=g^{-1}(K)$ pour un compact $K$ de cardinal au moins deux, alors il existe une rotation $R$ préservant $K$ telle que $f=R \circ g$. Nous avons déterminé dans [9] les polynômes $f, g$ et les compacts $K$ de capacité logarithmique positive vérifiant $f^{-1}(K)=g^{-1}(K)$. Des problèmes analogues pour les fonctions entières ou méromorphes ont été étudiés par Nevanlinna [28], Gross-Yang [21], Shiffman [33], etc. Ces auteurs utilisent des méthodes variées.

Ici, à partir de la relation $f^{-1}(K)=g^{-1}(K)$, on déduit que $g \circ f^{-1}(K)=K$ et par conséquent

$$
\left(g \circ f^{-1}\right) \circ \cdots \circ\left(g \circ f^{-1}\right)(K)=K,
$$

ce qui permet de se ramener à l'étude dynamique de la fonction multivaluée $F:=f \circ g^{-1}$ qui est en fait une correspondance polynomiale. Les propriétés dynamiques que nous allons étudier permettent de caractériser les ensembles d'unicité infinis (voir le corollaire 5.2).

Clozel et Ullmo étudient dans [7], [6] les correspondances holomorphes sur les surfaces de Riemann et sur les domaines symétriques. Ils en donnent des applications en arithmétique. Ils montrent que les correspondances modulaires sur une courbe holomorphe hyperbolique sont celles qui préservent la forme volume $\Omega$ associée à la métrique de Kobayashi. Ils en déduisent que les correspondances, qui commutent avec une correspondance modulaire extérieure, sont modulaires car ces correspondances, elles aussi, doivent préserver $\Omega$.

Depuis les travaux de Julia [24], Fatou [17], Ritt [31], Eremenko [16] (voir aussi [11]), on sait que si deux endomorphismes de $\mathbb{P}^{k}$ commutent, les objets dynamiques, qui leur sont associés, sont fortement liés. L'étude de ces objets permet de déterminer ou de caractériser ces endomorphismes. Dans le cadre des correspondances holomorphes, une étude dynamique devrait permettre de comprendre les commutateurs (voir le corollaire 2.9).

Nous renvoyons le lecteur à [2], [18], [19], [32], [34], pour les aspects fondamentaux de la théorie d'itération des applications holomorphes et méromorphes de $\mathbb{P}^{k}$. Pour les endomorphismes holomorphes de $\mathbb{P}^{k}$ ou pour les automorphismes de Hénon de $\mathbb{C}^{2}$ par exemple, on sait construire des mesures invariantes, mélangeantes qui maximisent l'entropie. Ces mesures d'équilibre sont obtenues comme intersections de courants invariants positifs fermés de bidegré $(1,1)$.

Briend-Duval [3], [4] ont montré que la mesure d'équilibre de tout endomorphisme holomorphe de degré $d \geq 2$ de $\mathbb{P}^{k}$ est limite de masses de Dirac portées par les points périodiques répulsifs. C'est aussi la limite de masses de Dirac portées par les préimages de tout point $z$ n'appartenant pas à un ensemble exceptionnel algébrique $\mathcal{E}$. Antérieurement, Fornæss-Sibony [19] avaient montré que $\mathcal{E}$ est pluripolaire. En dimension 1, ces résultats ont été démontrés par Brolin pour les polynômes [5], Lyubich [26] et Freire-Lopès-Mañé [20] pour les fractions rationnelles. Notons ici que l'étude des endomorphismes holomorphes de $\mathbb{P}^{k}$, peut se ramener à l'étude des endomorphismes polynomiaux. Il suffit de

TOME $133-2005-\mathrm{N}^{\mathrm{O}} 3$ 
considérer le relevé de ces applications à $\mathbb{C}^{k+1}$. Dans [13], l'auteur et Sibony ont construit pour les applications d'allure polynomiale une mesure invariante d'entropie maximale et généralisé les théorèmes de Briend-Duval pour de grandes familles de telles applications (en particulier pour les applications de degré topologique plus grand qu'un certain degré dynamique et pour les applications polynomiales dont l'exposant de Lojasiewicz est supérieur à 1).

Dans le présent travail, nous allons généraliser ces résultats aux correspondances polynomiales. Notre article s'organise de la manière suivante. Au paragraphe 2 nous définissons les correspondances polynomiales sur $\mathbb{C}^{k}$ et leurs exposants de Lojasiewicz à l'infini. Nous construisons la mesure d'équilibre $\mu$ associée à une correspondance polynomiale $F$ d'exposant de Lojasiewicz $\ell>1$. Cette mesure est $F^{*}$-invariante, « mélangeante » à vitesse exponentielle et ne charge pas les ensembles pluripolaires. Nous montrons aussi que toute correspondance polynomiale, qui commute avec $F$, préserve la mesure d'équilibre $\mu$ de $F$ (voir le corollaire 2.9). La construction de $\mu$ suit une méthode donnée dans [13] (méthode par résolution de $\mathrm{dd}^{\mathrm{c}}$ ); elle est aussi valable pour les correspondances d'allure polynomiale ou pour les itérations aléatoires (voir aussi [12]). Pour certaines correspondances, on peut construire un courant invariant $T$ positif fermé de bidegré $(1,1)$. Mais il est peu probable que la mesure $T^{k}$ (même lorsqu'elle est bien définie) soit invariante quand $k \geq 2$.

Dans le troisième paragraphe, en adaptant une méthode géométrique développée par Lyubich [26] et Briend-Duval [3], [4] Sibony et l'auteur [13], [10]), nous construisons, pour les petites boules centrées en un point générique, beaucoup de branches inverses dont on contrôle la taille. La mesure $\mu$ réflète la distribution des préimages de tout point $z$ qui n'appartient pas à un ensemble exceptionnel $\mathcal{E}$. En collaboration avec Charles Favre, nous montrons que $\mathcal{E}$ est l'orbite positive de $\mathcal{E}_{0}$ où $\mathcal{E}_{0}$ est le plus grand sous-ensemble algébrique propre de $\mathbb{C}^{k}$ invariant par $F^{-1}$. Le cas des applications polynomiales d'exposant de Lojasiewicz $\ell>1$ est traité dans [13], [14] (voir aussi [22], [12]). On obtient alors que l'ensemble $\mathcal{E}$ est algébrique. Dans ces cas la méthode algébrique s'applique sans difficulté. Pour les correspondances, nous rencontrons plusieurs difficultés techniques.

Dans le quatrième paragraphe, nous montrons en particulier que les points périodiques réguliers répulsifs de $F$ sont denses et équidistribués sur le support de $\mu$.

Les résultats obtenus sont encore valables dans un cadre plus général. Afin de simplifier les notations, nous préférons nous limiter au cas de l'espace complexe $\mathbb{C}^{k}$. Dans [12], [10], nous étendons cette étude aux itérations aléatoires des correspondances sur les variétés kählériennes compactes.

Une interprétation géométrique des résultats obtenus est donnée à la fin du paragraphe 4 (voir les corollaires $4.7,4.8$ ). Cette vision géométrique nous semble intéressante même pour les endomorphismes holomorphes de $\mathbb{P}^{k}$ et les 
automorphismes de Hénon de $\mathbb{C}^{2}$. Dans le dernier paragraphe, nous appliquons les résultats obtenus pour déterminer les ensembles d'unicité infinis, pour les polynômes d'une variable.

Signalons un travail récent de Claire Voisin [36] dans lequel elle étudie la nonhyperbolicité de variétés projectives en utilisant des correspondances (voir aussi l'exemple 3.11). Du point de vue dynamique, les correspondances considérées par Clozel-Ullmo et Claire Voisin sont plus proches des automorphismes holomorphes tandis que celles étudiées dans le présent article sont plutôt proches des endomorphismes holomorphes de $\mathbb{P}^{k}$. Un outil que nous avons développé récemment avec Sibony [15] permet d'étudier les correspondances de type automorphisme.

Dans la suite, $\mathrm{B}(z, r)$ et $\overline{\mathrm{B}}(z, r)$ désignent la boule ouverte et la boule fermée de centre $z$ et de rayon $r$. Les disques, les boules et le diamètre diam(.) d'un ensemble sont définis ou mesurés en métrique euclidienne. L'aire aire(.) d'un disque, la masse $\|$.$\| d'un courant, les normes \mathrm{L}^{2}$ et $\mathcal{C}^{2}$ d'une fonction sont mesurés en métrique de Fubini-Study. La notation $\delta_{z}$ désigne la masse de Dirac en $z$ et $\mathbf{1}_{S}$ désigne la fonction indicatrice de $S$. Les préimages d'un point $z$ de $F$ sont aussi les images de $z$ par la correspondance $\bar{F}$ adjointe à $F$. Nous préférons parler de préimages plutôt que d'images afin que les applications polynomiales soient couvertes par notre étude.

Remerciements. - Je remercie Charles Favre et Nessim Sibony dont les nombreuses remarques ont permis d'améliorer la rédaction de cet article.

\section{Correspondances polynomiales}

Soit $X$ une variété complexe de dimension $k \geq 1$. Notons $\pi_{1}, \pi_{2}$ les projections canoniques de $X \times X$ dans $X$. On appelle $k$-chaîne holomorphe de $X \times X$ toute combinaison finie $Y:=\sum n_{i} Y_{i}$ où les $Y_{i}$ sont des sous-ensembles analytiques irréductibles de dimension $k$, deux à deux distincts, de $X \times X$ et où les $n_{i}$ sont des entiers relatifs non nuls. On dira que $Y$ est positive si les $n_{i}$ sont positifs. D'après un théorème de Lelong, une $k$-chaîne holomorphe positive $Y$ définit par intégration un courant positif fermé $[Y]$ de bidimension $(k, k)$ de $X \times X$. Notons $|Y|:=\bigcup Y_{i}$ le support de $Y$ et $\bar{Y}:=\sum n_{i} \bar{Y}_{i}$ où $\bar{Y}_{i}$ est le symétrique de $Y_{i}$ par rapport à la diagonale de $X \times X$, i.e. l'image de $Y_{i}$ par l'application $(x, y) \mapsto(y, x)$.

Une correspondance holomorphe de degré topologique $\left(d_{1}, d_{2}\right)$ sur $X$ est la donnée d'une $k$-chaîne holomorphe positive $Y$ de dimension $k$ de $X \times X$ telle que la restriction de $\pi_{i}$ à $Y$ définisse une application propre de degré $d_{i}$ pour $i=1,2$. Plus précisément, pour tout $z \in X$ la fibre $Y \cap \pi_{i}^{-1}(z)$ contient exactement $d_{i}$ points comptés avec multiplicités. Il est clair que si $(x, y) \in|Y|$ et si $x$ tend vers l'infini alors $y$ tend aussi vers l'infini et réciproquement. On peut identifier cette correspondance à la fonction multivaluée $F:=\left(\pi_{2 \mid Y}\right) \circ\left(\pi_{1 \mid Y}\right)^{-1}$. Le terme 
correspondance désignera $F$. On dira que $Y$ est le graphe de $F$. On utilisera souvent la décomposition $Y=\sum Y_{i}^{*}$ dans laquelle chaque $Y_{i}$ est répété $n_{i}$ fois afin d'éviter de parler de multiplicités. La correspondance $\bar{F}$ associée à $\bar{Y}$ est appelée correspondance adjointe de $F$.

Soit $F^{\prime}$ une autre correspondance de degré topologique $\left(d_{1}^{\prime}, d_{2}^{\prime}\right)$ associée à une $k$-chaîne holomorphe positive $Z=\sum Z_{j}^{*}$. La composition $F^{\prime} \circ F$ est celle associée au produit fibré $Y \times_{X} Z:=\sum\left(Y_{i}^{*} \times_{X} Z_{j}^{*}\right)$ où

$$
\begin{aligned}
Y_{i}^{*} \times{ }_{X} Z_{j}^{*}:=\{(x, z) \in X \times X \text { tel qu'il existe } & \\
& \left.y \in X \text { vérifiant }(x, y) \in Y_{i}^{*} \text { et }(y, z) \in Z_{j}^{*}\right\} .
\end{aligned}
$$

Le produit $Y_{i}^{*} \times_{X} Z_{j}^{*}$ est, en général, une $k$-chaîne holomorphe qui n'est pas toujours irréductible. Dans la formule précédente le nombre des valeurs convenables de $y$ détermine les multiplicités. La composition $F^{\prime} \circ F$ est une correspondance de degré topologique $\left(d_{1} d_{1}^{\prime}, d_{2} d_{2}^{\prime}\right)$. On notera $F^{n}$ la correspondance $F \circ \cdots \circ F$ ( $n$ fois).

Dans le présent travail, nous considérons le cas où $X$ est l'espace euclidien $\mathbb{C}^{k}$ et les composantes $Y_{i}$ de $Y$ sont des sous-ensembles algébriques de $\mathbb{C}^{k} \times \mathbb{C}^{k}$. On dira qu'une telle correspondance $F$ est polynomiale (propre). Il existe une constante $\ell>0$ telle que pour tout $(x, y) \in|Y|$ suffisamment grand on ait $|y| \geq c|x|^{\ell}$ où $c>0$ est une constante. Si $F$ est un endomorphisme polynomial, Ploski [30] a montré qu'il existe une constante maximale $\ell>0$ qui vérifie la propriété ci-dessus. Sa preuve est aussi valable pour les correspondances polynomiales. Cette constante $\ell$ est appelée exposant de Lojasiewicz de $F$. Dans la suite, on suppose que $\ell>1$. On vérifie que dans ce cas $d_{1}$ est strictement plus petit que $d_{2}$ (on peut prouver ceci en utilisant l'argument donné dans la proposition 4.1). Notons $z$ les coordonnées euclidiennes de $\mathbb{C}^{k}$ et

$$
\omega:=\frac{1}{2} \mathrm{dd}^{\mathrm{c}} \log \left(1+\|z\|^{2}\right)
$$

la forme de Fubini-Study de $\mathbb{P}^{k}$. Soit $A_{0}>1$ une constante assez grande que nous allons choisir dans le lemme 2.5. Fixons un nombre $R_{0}>0$ assez grand tel que $|y|>A_{0}|x|$ pour tout $(x, y) \in|Y|$ vérifiant $|y| \geq R_{0}$. On pose

$$
\begin{gathered}
F^{-1}:=\left(\pi_{1 \mid Y}\right) \circ\left(\pi_{2 \mid Y}\right)^{-1} \\
F^{*}:=\left(\pi_{1 \mid Y}\right)_{*}\left(\pi_{2 \mid Y}\right)^{*}, \quad F_{*}:=(\bar{F})^{*}=\left(\pi_{2 \mid Y}\right)_{*}\left(\pi_{1 \mid Y}\right)^{*} .
\end{gathered}
$$

Les « applications » $F$ et $F^{-1}$ agissent sur les sous-ensembles de $\mathbb{C}^{k}$, les points de la fibre $F^{-1}(z)$ de $F$ sont comptés avec multiplicités. L'opérateur $F_{*}$ agit sur les fonctions continues ou plurisousharmoniques (p.s.h.) et sur les courants positifs fermés de bidegré $(1,1)$ de $\mathbb{C}^{k}$. L'opérateur $F^{*}$ agit sur les mesures positives. Plus précisément, si $\varphi$ est une fonction continue ou p.s.h. sur $\mathbb{C}^{k}$, on pose

$$
F_{*} \varphi:=\sum_{w \in F^{-1}(z)} \varphi(w) .
$$

BULletin DE LA SOCiÉtÉ MATHÉMATIQUE DE FRANCE 
C'est une fonction continue ou p.s.h. sur $\mathbb{C}^{k}$. Rappelons que $\varphi$ est p.s.h. si elle

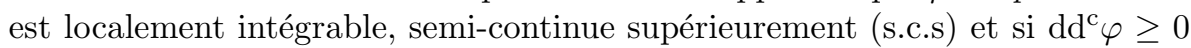
au sens des courants. Elle est pluriharmonique si elle est continue et $\operatorname{dd}^{\mathrm{c}} \varphi=0$. Observons que si $\varphi$ est pluriharmonique, $F_{*} \varphi$ l'est aussi. Si $T$ est un courant positif fermé de bidegré $(1,1)$ sur $\mathbb{C}^{k}$, il existe une fonction p.s.h. $\varphi$, unique à une fonction pluriharmonique près, telle que $\operatorname{dd}^{\mathrm{c}} \varphi=T$. On définit alors $F_{*} T:=\mathrm{dd}^{\mathrm{c}} F_{*} \varphi$ (voir [27]). Pour une mesure positive $\nu$ à support compact sur $\mathbb{C}^{k}$, on définit $F^{*} \nu$ par

$$
\left\langle F^{*} \nu, \varphi\right\rangle:=\left\langle\nu, F_{*} \varphi\right\rangle \text { pour } \varphi \text { continue sur } \mathbb{C}^{k} .
$$

Nous dirons qu'une fonction p.s.h. $\varphi$ sur $\mathbb{C}^{k}$ est à croissance logarithmique s'il existe une constante $A>0$ telle que $\varphi-A \log \left(1+\|z\|^{2}\right)$ soit bornée supérieurement. On dit qu'une mesure positive est $P B$ si elle intègre les fonctions p.s.h. à croissance logarithmique [13]. Dans le cas de dimension $k=1$, si $\mu$ est une mesure positive sur $\mathbb{C}$, on peut écrire $\mu=\operatorname{dd}^{\mathrm{c}} u-\alpha$ avec $u$ une fonction $\mathrm{L}^{1}$ et $\alpha$ une forme lisse sur $\mathbb{P}^{1}$. On a montré [13] que $\mu$ est PB si et seulement si son potentiel $u$ est borné. Ceci justifie la terminologie choisie. D'après le théorème de Josefson [25, th. 5.2.4], pour tout ensemble pluripolaire $E$, il existe une fonction p.s.h. à croissance logarithmique $\varphi$ telle que $\varphi=-\infty$ sur $E$. Par conséquent, les mesures $\mathrm{PB}$ ne chargent pas les ensembles pluripolaires. En particulier, le support d'une mesure $\mathrm{PB}$ est parfait, i.e. ne contient pas de point isolé.

ThÉORÈme 2.1. - Soit F une correspondance polynomiale de degré topologique $\left(d_{1}, d_{2}\right)$ sur $\mathbb{C}^{k}$, d'exposant de Lojasiewicz $\ell>1$. Soient $\nu_{n}$ des mesures de probabilité de support uniformément borné dans $\mathbb{C}^{k}$. Supposons que $\nu_{n}=h_{n} \omega^{k}$ où $h_{n}$ est une fonction vérifiant $\left\|h_{n}\right\|_{\mathrm{L}^{2}}=\mathrm{o}\left(\ell^{n}\right)$. Alors la suite de mesures $d_{2}^{-n}\left(F^{n}\right)^{*} \nu_{n}$ converge vers une mesure de probabilité $\mu$, à support compact, indépendante de la suite $\left(\nu_{n}\right)$. De plus, cette mesure est $P B$ et vérifie la relation de $F^{*}$-invariance $F^{*} \mu=d_{2} \mu$.

On dit que $\mu$ est la mesure d'équilibre de $F$. En général, elle n'est pas invariante par $F$, i.e. $F_{*} \mu \neq d_{1} \mu$. On a $\mu=\lim d_{2}^{-n}\left(F^{n}\right)^{*} \omega^{k}$. Pour montrer le théorème 2.1 , l'idée est de tester une fonction p.s.h. $\varphi$. On a

$$
d_{2}^{-n}\left\langle\left(F^{n}\right)^{*}\left(\nu_{n}\right), \varphi\right\rangle=\left\langle\nu_{n}, d_{2}^{-n}\left(F^{n}\right)_{*} \varphi\right\rangle .
$$

Nous allons montrer que $d_{2}^{-n}\left(F^{n}\right)_{*} \varphi$ tend dans $\mathrm{L}_{\text {loc }}^{2}$ à vitesse $\mathrm{O}\left(\ell^{-n}\right)$ vers une constant $c_{\varphi}$. Le théorème en découle.

Fixons une boule $V:=\mathrm{B}(0, R)$ de rayon $R>R_{0}$ qui contient les supports des $\nu_{n}$. Posons $U:=F^{-1}(V)$. Alors $\bar{U}$ est contenu dans la boule $U^{\prime}:=\mathrm{B}\left(0, R / A_{0}\right)$. Fixons aussi $V^{\prime}:=\mathrm{B}\left(0, R^{\prime}\right)$ avec $R^{\prime}>R$ tel que $F^{-1}\left(V^{\prime}\right) \subset U^{\prime}$. Soit $\varphi$ une fonction sur $V$ ou sur $\mathbb{C}^{k}$. Soit $\Lambda:=d_{2}^{-1} F_{*}$ l'opérateur de Perron-Frobenius 
associé à $F$. On a par définition

$$
\Lambda \varphi(z)=d_{2}^{-1} \sum_{w \in F^{-1}(z)} \varphi(w)
$$

où les points de $F^{-1}(z)$ sont comptés avec multiplicités. Posons $\varphi_{n}:=\Lambda^{n} \varphi$. Cette fonction est p.s.h. ou continue si $\varphi$ l'est. Nous aurons besoin des lemmes suivants.

LEMme 2.2. - Soit $T$ un courant positif fermé de bidegré $(1,1)$ et de masse 1 sur $\mathbb{P}^{k}$. Alors la masse de $\Lambda^{n} T$ dans $\mathbb{C}^{k}$ est plus petite ou égale à $\ell^{-n}$.

Démonstration. - Dans $\mathbb{C}^{k}$, on peut écrire $T=\operatorname{dd}^{\mathrm{c}} \varphi$ où $\varphi$ est une fonction p.s.h. telle que $\varphi-\frac{1}{2} \log \left(1+\|z\|^{2}\right)$ soit bornée supérieurement. On a

$$
\ell^{n} \Lambda^{n} T=\operatorname{dd}^{\mathrm{c}}\left(\ell^{n} \Lambda^{n} \varphi\right)=\operatorname{dd}^{\mathrm{c}}\left(\ell^{n} \varphi_{n}\right) .
$$

Comme l'exposant de Lojasiewicz de $F$ est égal à $\ell>1$ on peut, quitte à effectuer un changement linéaire de coordonnées, supposer que $\left\|z^{\prime}\right\|^{\ell} \leq\|z\|$ lorsque $\|z\|$ est assez grand et $z^{\prime} \in F^{-1}(z)$. La fonction $\ell^{n} \varphi_{n}-\frac{1}{2} \log \left(1+\|z\|^{2}\right)$ est donc bornée supérieurement car les valeurs de $\varphi_{n}(z)$ sont obtenues comme la moyenne des valeurs de $\varphi$ sur $F^{-n}(z)$. D'après un lemme de comparaison (voir par exemple [34], [14, prop. 5.4]), ceci implique que

$$
\begin{aligned}
\left\|\ell^{n} \Lambda^{n} T\right\|_{\mathbb{C}^{k}} & =\int_{\mathbb{C}^{k}} \operatorname{dd}^{\mathrm{c}}\left(\ell^{n} \varphi_{n}\right) \wedge \omega^{k-1} \\
& \leq \frac{1}{2} \int_{\mathbb{C}^{k}} \mathrm{dd}^{\mathrm{c}} \log \left(1+\|z\|^{2}\right) \wedge \omega^{k-1}=\int_{\mathbb{C}^{k}} \omega^{k}=1 .
\end{aligned}
$$

LEMME 2.3. - Soit $\varphi$ une fonction p.s.h. sur $V$ telle que $\varphi_{n}:=\Lambda^{n} \varphi$ ne tende pas uniformément vers $-\infty$ (en particulier si $\varphi$ est localement bornée). Alors $\varphi_{n}$ tend vers une constante $c_{\varphi}$ dans l'espace $\mathrm{L}_{\text {loc }}^{2}\left(\mathbb{C}^{k}\right)$.

Démonstration. - Observons que $\varphi_{n}$ est définie dans la boule $\mathrm{B}\left(0, A_{0}^{n} R\right)$. On montre que $\varphi_{n}$ tend vers une constante $c_{\varphi}$ dans $\mathrm{L}_{\text {loc }}^{2}(V)$. Pour la convergence dans $\mathrm{L}_{\text {loc }}^{2}\left(\mathbb{C}^{k}\right)$ il suffit de remplacer $V$ par des boules suffisamment grandes.

Soit $\psi$ la régularisée s.c.s. de la fonction $\left(\lim \sup \varphi_{n}\right)$ sur $V$. C'est une fonction p.s.h. car $\varphi_{n}$ ne tend pas uniformément vers $-\infty$. Comme $F^{-1}(V)=U$,

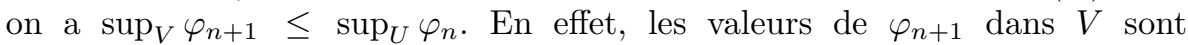
obtenues comme la moyenne de valeurs de $\varphi_{n}$ dans $U$. Ceci implique que $\sup _{V} \psi \leq \sup _{U} \psi$ car $\psi$ est aussi égale à la régularisée s.c.s. de la fonction $\left(\lim \sup \varphi_{n+1}\right)$. Le principe du maximum entraîne que $\psi$ est une constante $c_{\varphi}$.

Soit $\left(\varphi_{n_{i}}\right)$ une sous-suite convergente dans $\mathrm{L}_{\text {loc }}^{2}(V)$ vers une fonction p.s.h. $h$. Montrons que $h=c_{\varphi}$. On a $h \leq c_{\varphi}$. Si $h \neq c_{\varphi}$, le principe du maximum implique que $h \leq c_{\varphi}-2 \varepsilon$ sur $U^{\prime}$ où $\varepsilon>0$ est une constante. D'après le lemme de Hartogs [23, th. 2.6.4], on a $\varphi_{n_{i}} \leq c_{\varphi}-\varepsilon$ sur $U$ pour $i$ assez grand. Par conséquent, $\varphi_{n} \leq c_{\varphi}-\varepsilon$ sur $V$ pour tout $n>n_{i}$. Ceci contredit le fait que la 
régularisée s.c.s. de $\left(\lim \sup \varphi_{n}\right)$ est égale à $c_{\varphi}$. On a montré que $\varphi_{n}$ tend vers $c_{\varphi}$ dans $\mathrm{L}_{\text {loc }}^{2}(V)$.

Le lemme précédent permet de construire la mesure d'équilibre $\mu$. Si $\varphi$ est une fonction de classe $\mathcal{C}^{2}$ à support compact, la suite $\varphi_{n}$ converge aussi vers une constante $c_{\varphi}$ dans $\mathrm{L}_{\text {loc }}^{2}\left(\mathbb{C}^{k}\right)$ car cette fonction $\varphi$ s'écrit comme différence de deux fonctions p.s.h. Si $\Omega$ est une forme volume lisse à support dans $V$ telle que $\int \Omega=1$, on a d'après le lemme 2.3

$$
\lim _{n \rightarrow \infty}\left\langle d_{2}^{-n}\left(F^{n}\right)^{*} \Omega, \varphi\right\rangle=\lim _{n \rightarrow \infty}\left\langle\Omega, \Lambda^{n} \varphi\right\rangle=c_{\varphi} .
$$

Par conséquent, $d_{2}^{-n}\left(F^{n}\right)^{*} \Omega$ tend faiblement vers une mesure de probabilité $\mu$ définie par $\langle\mu, \varphi\rangle:=c_{\varphi}$ pour $\varphi$ de classe $\mathcal{C}^{2}$. Il est clair que $\mu$ est portée par $\bar{U}$ et vérifie la relation de $F^{*}$-invariance. Elle ne dépend pas de $\Omega$. Observons ici que la fonction p.s.h. $\varphi$ vérifie l'hypothèse du lemme 2.3 si et seulement si elle est $\mu$-intégrable.

LEMME 2.4. - Soit $\varphi$ une fonction p.s.h. sur $V$. Si $\varphi$ n'est pas $\mu$-intégrable, la suite de fonctions $\varphi_{n}:=\Lambda^{n} \varphi$ converge uniformément vers $-\infty$. Si $\varphi$ est $\mu$-intégrable, $\left(\varphi_{n}\right)$ converge dans $\mathrm{L}_{\text {loc }}^{2}\left(\mathbb{C}^{k}\right)$ vers la constante $c_{\varphi}:=\int \varphi \mathrm{d} \mu$.

Démonstration. - Comme $\mu$ est $F^{*}$-invariante, on a $\langle\mu, \varphi\rangle=\left\langle\mu, \varphi_{n}\right\rangle$ pour tout $n \geq 0$. D'après le lemme 2.3 , il suffit de traiter le cas où la suite $\left(\varphi_{n}\right)$ converge dans $\mathrm{L}_{\text {loc }}^{2}\left(\mathbb{C}^{k}\right)$ vers une constante $c_{\varphi}$. Montrons que $c_{\varphi}=\int \varphi \mathrm{d} \mu$.

D'après le lemme de Hartogs (voir le lemme 2.3), on a $\lim \sup \varphi_{n}=c_{\varphi}$. On déduit de la relation $\langle\mu, \varphi\rangle=\left\langle\mu, \varphi_{n}\right\rangle$ que $\langle\mu, \varphi\rangle \leq c_{\varphi}$. D'autre part, la semi-continuité supérieure de $\varphi$ et la définition de $\mu$ impliquent que

$$
\langle\mu, \varphi\rangle \geq \lim \sup \left\langle d_{t}^{-n}\left(F^{n}\right)^{*} \Omega, \varphi\right\rangle=\lim \sup \left\langle\Omega, \varphi_{n}\right\rangle=c_{\varphi} .
$$

La preuve du lemme est achevée.

LEMme 2.5. - Soit $\varphi$ une fonction pluriharmonique sur $V$. Alors pour $A_{0}$ assez grand, on a

$$
\left\|\varphi_{n}-c_{\varphi}\right\|_{\mathrm{L}^{\infty}(V)} \leq 2^{-n} \ell^{-n}\left\|\varphi-c_{\varphi}\right\|_{\mathrm{L}^{\infty}(V)} .
$$

Démonstration. - Quitte à remplacer $\varphi$ par $\varphi-c_{\varphi}$, on peut supposer que $c_{\varphi}=\langle\mu, \varphi\rangle=0$. Le support de $\mu$ étant contenu dans la boule $U^{\prime}=\mathrm{B}\left(0, R / A_{0}\right)$, l'égalité $\langle\mu, \varphi\rangle=0$ implique que $\varphi$ doit s'annuler en un point de cette boule. D'après un lemme du type Schwarz, on a

$$
\|\varphi\|_{\mathrm{L}^{\infty}(U)} \leq(2 \ell)^{-1}\|\varphi\|_{\mathrm{L}^{\infty}(V)}
$$

lorsque $A_{0}$ est assez grand. Par conséquent, $\left\|\varphi_{1}\right\|_{\mathrm{L}^{\infty}(V)} \leq(2 \ell)^{-1}\|\varphi\|_{\mathrm{L}^{\infty}(V)}$. Ceci implique le lemme. Notons que pour montrer le lemme du type Schwarz, on peut considérer la famille normale des fonctions harmoniques $\psi$ sur le disque unité $\Delta \subset \mathbb{C}$ s'annulant en un point de $\Delta\left(0,1 / A_{0}\right)$ et vérifiant $\psi \leq 1$.

TOME $133-2005-\mathrm{N}^{\mathrm{O}} 3$ 
Notons $\operatorname{PSH}(V)$ le cône des fonctions p.s.h. sur $V$. Rappelons que les boules $U^{\prime}$ et $V^{\prime}$ sont fixées au début de la démonstration du théorème 2.1.

Lemme 2.6. - L'opérateur $\Lambda: \operatorname{PSH}\left(U^{\prime}\right) \cap \mathrm{L}^{2}\left(U^{\prime}\right) \longrightarrow \operatorname{PSH}(V) \cap \mathrm{L}^{2}(V)$ est borné dans le sens où il existe une constante $c>0$ telle que

$$
\|\Lambda \varphi\|_{\mathrm{L}^{2}(V)} \leq c\|\varphi\|_{\mathrm{L}^{2}\left(U^{\prime}\right)}
$$

pour toute fonction $\varphi \in \operatorname{PSH}\left(U^{\prime}\right) \cap \mathrm{L}^{2}\left(U^{\prime}\right)$.

Démonstration. - Soit $\varphi^{(n)}$ une suite de fonctions p.s.h. sur $U^{\prime}$ telle que $\left\|\varphi^{(n)}\right\|_{L^{2}\left(U^{\prime}\right)} \leq 1$. Il faut montrer que la suite $\Lambda \varphi^{(n)}$ est bornée dans $L^{2}(V)$.

Quitte à extraire une sous-suite, on peut supposer que la suite $\varphi^{(n)}$ converge dans $\mathrm{L}_{\text {loc }}^{2}\left(U^{\prime}\right)$ vers une fonction p.s.h. $\varphi$. On en déduit que la suite de fonctions p.s.h. $\Lambda \varphi^{(n)}$ converge vers $\Lambda \varphi$ dans $\mathrm{L}_{\text {loc }}^{2}\left(V^{\prime}\right)$. En particulier, elle converge vers $\Lambda \varphi$ dans $\mathrm{L}^{2}(V)$.

Notons $\mathrm{PH}(V)$ l'espace des fonctions pluriharmoniques sur $V$. Posons

$$
H:=\mathrm{PH}(V) \cap \mathrm{L}^{2}(V)
$$

et $H^{\perp}$ son orthogonal dans $\mathrm{L}^{2}(V)$. Posons aussi

$$
H^{\perp *}:=\operatorname{PSH}(V) \cap H^{\perp} .
$$

Le sous-espace $H$ est invariant par $\Lambda$ car si $\varphi$ est pluriharmonique sur $V, \Lambda \varphi$ l'est sur un voisinage de $\bar{V}$. Pour toute fonction $\varphi \in \operatorname{PSH}\left(V^{\prime}\right)$, on a la décomposition unique $\varphi=u+v$ avec $u \in H$ et $v \in H^{\perp *}$. La fonction $v$ est le potentiel dans $V$ du courant $\operatorname{dd}^{\mathrm{c}} \varphi$ dont la norme $\mathrm{L}^{2}$ est minimale.

D'après le lemme 2.6, l'opérateur

$$
\Lambda: \operatorname{PSH}(V) \cap \mathrm{L}^{2}(V) \longrightarrow \operatorname{PSH}(V) \cap \mathrm{L}^{2}(V)
$$

est borné. Par conséquent, il existe des applications linéaires bornées

$$
\Lambda_{1}: H \longrightarrow H, \quad \Lambda_{2}: H^{\perp *} \longrightarrow H, \quad \Lambda_{3}: H^{\perp *} \longrightarrow H^{\perp *}
$$

telles que $\Lambda \varphi=\Lambda_{1} u+\Lambda_{2} v+\Lambda_{3} v$. On a $\Lambda_{1}=\Lambda_{\mid H}$ et $\Lambda_{2}=\operatorname{pr}_{H} \circ \Lambda_{\mid H^{\perp *}}$ et $\Lambda_{3}=\operatorname{pr}_{H^{\perp}} \circ \Lambda_{\mid H^{\perp *}}$ où $\operatorname{pr}_{\mid H}$ et $\operatorname{pr}_{\mid H^{\perp}}$ désignent les projections orthogonales de $\mathrm{L}^{2}(V)$ sur $H$ et sur $H^{\perp}$. On a $\operatorname{dd}^{\mathrm{c}} \Lambda_{3}^{n} \varphi=\operatorname{dd}^{\mathrm{c}} \varphi_{n}$.

Proposition 2.7. - La mesure $\mu$ est PB. De plus, il existe une constante $c>0$ telle que l'on ait

$$
\left\|\Lambda^{n} \varphi-c_{\varphi}\right\|_{L^{2}(V)} \leq c A \ell^{-n}
$$

pour toute fonction p.s.h. $\varphi$ avec $\|\varphi\|_{\mathrm{L}^{2}(V)} \leq A$ et $\varphi-\frac{1}{2} A \log \left(1+\|z\|^{2}\right)$ bornée supérieurement et pour tout $n \geq 1$. 
Démonstration. - Par homothétie, il suffit de considérer une fonction $\varphi$ p.s.h. avec $\|\varphi\|_{\mathrm{L}^{2}(V)} \leq 1$ et $\varphi-\frac{1}{2} \log \left(1+\|z\|^{2}\right)$ bornée supérieurement. La famille de telles fonctions est compacte. Les constantes $c_{i}$ et $c$ que nous allons utiliser sont positives et indépendantes de $\varphi$. D'après le lemme 2.2 , on a $\left\|\operatorname{dd}^{\mathrm{c}} \varphi_{n}\right\| \leq \ell^{-n}$. Par conséquent, il existe une fonction p.s.h. $\psi_{n}$ telle que $\psi_{n}-\frac{1}{2} \ell^{-n} \log \left(1+\|z\|^{2}\right)$ soit bornée supérieurement, $\left\|\psi_{n}\right\|_{\mathrm{L}^{2}} \leq c_{1} \ell^{-n}$ et $\mathrm{dd}^{\mathrm{c}} \psi_{n}=\operatorname{dd}^{\mathrm{c}} \varphi_{n}$. Il s'agit ici la résolution de $\mathrm{dd}^{\mathrm{c}}$ sur $\mathbb{P}^{k}$. On en déduit que $\left\|\Lambda_{3}^{n} v\right\|_{\mathrm{L}^{2}(V)} \leq\left\|\psi_{n}\right\|_{\mathrm{L}^{2}} \leq c_{1} \ell^{-n}$. Posons

$$
b:=\int u \mathrm{~d} \mu, \quad b_{n}:=\int \Lambda_{2} \Lambda_{3}^{n} v \mathrm{~d} \mu, \quad s_{n}:=b+b_{0}+\cdots+b_{n-1} .
$$

La fonction pluriharmonique $u$ vérifie $\|u\|_{\mathrm{L}^{2}(V)} \leq\|\varphi\|_{\mathrm{L}^{2}(V)} \leq 1$, on en déduit que $\|u\|_{L^{\infty}(U)} \leq c_{2}$ par la formule de la moyenne. Comme $\Lambda_{2}$ est borné, on a

$$
\left\|\Lambda_{2} \Lambda_{3}^{n} v\right\|_{\mathrm{L}^{2}(V)} \leq c_{3}\left\|\Lambda_{3}^{n} v\right\|_{\mathrm{L}^{2}(V)} \leq c_{1} c_{3} \ell^{-n} .
$$

La formule de la moyenne appliquée à la fonction pluriharmonique $\Lambda_{2} \Lambda_{3}^{n} v$ implique que $\left|b_{n}\right| \leq c_{4} \ell^{-n}$ et donc la suite $\left(s_{n}\right)$ est convergente. Utilisant les inégalités obtenues et le lemme 2.5 et l'estimation de $b_{j}$, on obtient en développant $\Lambda^{n}$,

$$
\begin{gathered}
\left\|\Lambda^{n} \varphi-s_{n}\right\|_{\mathrm{L}^{2}(V)}=\| \Lambda_{1}^{n} u+\Lambda_{1}^{n-1} \Lambda_{2} v+\Lambda_{1}^{n-2} \Lambda_{2} \Lambda_{3} v+\cdots+\Lambda_{1} \Lambda_{2} \Lambda_{3}^{n-2} v \\
+\Lambda_{2} \Lambda_{3}^{n-1} v-s_{n}\left\|_{\mathrm{L}^{2}(V)}+\right\| \Lambda_{3}^{n} v \|_{\mathrm{L}^{2}(V)} \\
\leq\left\|\Lambda_{1}^{n} u-b\right\|_{\mathrm{L}^{2}(V)}+\left\|\Lambda_{1}^{n-1} \Lambda_{2} v-b_{0}\right\|_{\mathrm{L}^{2}(V)} \\
+\left\|\Lambda_{1}^{n-2} \Lambda_{2} \Lambda_{3} v-b_{1}\right\|+\cdots+\left\|\Lambda_{1} \Lambda_{2} \Lambda_{3}^{n-2} v-b_{n-2}\right\| \\
+\left\|\Lambda_{2} \Lambda_{3}^{n-1} v-b_{n-1}\right\|_{\mathrm{L}^{2}(V)}+\left\|\Lambda_{3}^{n} v\right\|_{\mathrm{L}^{2}(V)} \\
\leq c_{5}\left[(2 \ell)^{-n}+(2 \ell)^{-n+1}+(2 \ell)^{-n+2} \ell^{-1}\right. \\
\left.+\cdots+(2 \ell)^{-1} \ell^{-n+2}+\ell^{-n+1}+\ell^{-n}\right] \leq c_{6} \ell^{-n}
\end{gathered}
$$

On en déduit que $\Lambda^{n} \varphi$ converge vers la constante $\lim s_{n}=b+\sum b_{i}$. Par conséquent, $\varphi$ est $\mu$-intégrable et $c_{\varphi}=b+\sum b_{i}$. On a aussi $\left|c_{\varphi}-s_{n}\right| \leq c_{7} \ell^{-n}$. Ceci implique que $\left\|\Lambda^{n} \varphi-c_{\varphi}\right\|_{\mathrm{L}^{2}(V)} \leq c \ell^{-n}$.

Corollaire 2.8. - Il existe une constante $c>0$ telle que

$$
\sup _{V}\left(\varphi_{n}-c_{\varphi}\right) \leq c A \ell^{-n}
$$

pour tout $n \geq 1$ et toute fonction $\varphi$ p.s.h. sur $\mathbb{C}^{k}$ avec $\|\varphi\|_{\mathrm{L}^{2}\left(V^{\prime}\right)} \leq A$ et $\varphi-\frac{1}{2} A \log \left(1+\|z\|^{2}\right)$ bornée supérieurement.

Démonstration. - Il suffit d'appliquer la proposition 2.7 en remplaçant $V$ par une boule $V_{1}$ telle que $\bar{V} \subset V_{1}$ et $\bar{V}_{1} \subset V^{\prime}$, puis d'utiliser l'inégalité de la sous-moyenne pour les fonctions p.s.h.

TOME $133-2005-\mathrm{N}^{\mathrm{O}} 3$ 
Fin de la démonstration du théorème 2.1. — Soit $\varphi$ une fonction p.s.h. à croissance logarithmique. D'après l'inégalité de Cauchy-Schwarz, on a

$$
\begin{aligned}
\left|\left\langle d_{2}^{-n}\left(F^{n}\right)^{*} \nu_{n}-\mu, \varphi\right\rangle\right| & =\left|\left\langle\nu_{n}, \Lambda^{n} \varphi\right\rangle-c_{\varphi}\right|=\left|\left\langle h_{n} \omega^{k}, \Lambda^{n} \varphi-c_{\varphi}\right\rangle\right| \\
& \leq\left\|h_{n}\right\|_{L^{2}(V)}\left\|\varphi_{n}-c_{\varphi}\right\|_{L^{2}(V)} .
\end{aligned}
$$

D'après la proposition 2.7, le majorant tend vers 0 car $\left\|h_{n}\right\|_{\mathrm{L}^{2}(V)}=\mathrm{o}\left(\ell^{n}\right)$. On en déduit que $d_{2}^{-n}\left(F^{n}\right)^{*} \nu_{n}$ tend faiblement vers $\mu$.

Corollaire 2.9. - Soit F une correspondance comme au théorème 2.1. Si G est une autre correspondance polynomiale de degré topologique $\left(d_{1}^{\prime}, d_{2}^{\prime}\right)$, d'exposant de Lojasiewicz quelconque et vérifiant $F \circ G=G \circ F$ alors $G^{*} \mu=d_{2}^{\prime} \mu$.

Démonstration. - Soit $\nu$ une forme volume lisse à support compact sur $\mathbb{C}^{k}$ telle que $\int_{\mathbb{C}^{k}} \nu=1$. D'après le théorème 2.1 , on a $\lim d_{2}^{-n}\left(F^{n}\right)^{*} \nu=\mu$ et $\lim d_{2}^{-n}\left(F^{n}\right)^{*}\left(G^{*} \nu\right)=d_{2}^{\prime} \mu$. Du fait que $F$ et $G$ commutent, on a

$$
\lim _{n \rightarrow \infty} d_{2}^{-n}\left(F^{n}\right)^{*}\left(G^{*} \nu\right)=\lim _{n \rightarrow \infty} d_{2}^{-n} G^{*}\left(F^{n}\right)^{*} \nu=G^{*} \mu .
$$

On en déduit que $G^{*} \mu=d_{2}^{\prime} \mu$.

THÉORÈmE 2.10. - Soit F une correspondance comme au théorème 2.1. Alors la mesure $\mu$ est $F^{*}$-mélangeante d'ordre exponentiel. Plus précisément, il existe une constante $c>0$ telle que pour toute fonction $\varphi$ de classe $\mathcal{C}^{2}$ et toute fonction $\psi$ bornée sur $\mathbb{C}^{k}$, on ait

$$
\left|I_{n}(\varphi, \psi)\right| \leq c \ell^{-n}\|\varphi\|_{\mathcal{C}^{2}} \cdot\|\psi\|_{\mathrm{L}^{\infty}}
$$

où

$$
I_{n}(\varphi, \psi):=\int\left(\Lambda^{n} \varphi\right) \psi \mathrm{d} \mu-\left(\int \varphi \mathrm{d} \mu\right)\left(\int \psi \mathrm{d} \mu\right)
$$

Démonstration. - Nous allons d'abord considérer le cas où $\varphi$ est p.s.h. avec $\|\varphi\|_{\mathrm{L}^{2}\left(V^{\prime}\right)} \leq A$ et $\varphi-\frac{1}{2} A \log \left(1+\|z\|^{2}\right)$ bornée supérieurement. Montrons que $I_{n}(\varphi, \psi) \leq c A \ell^{-n}\|\psi\|_{L^{\infty}}$. Observons que l'on a $I_{n}(\varphi, \psi+\alpha)=I_{n}(\varphi, \psi)$ si $\alpha$ est une constante, car $\mu$ est $F^{*}$-invariante. Il suffit donc d'examiner le cas où $\psi$ est positive. On a d'après le corollaire 2.8

$$
I_{n}(\varphi, \psi)=\int\left(\Lambda^{n} \varphi-c_{\varphi}\right) \psi \mathrm{d} \mu \leq \int c A \ell^{-n} \psi \mathrm{d} \mu \leq c A \ell^{-n}\|\psi\|_{L^{\infty}} .
$$

De la même manière, on montre que $-I_{n}(\varphi, \psi)=I_{n}(\varphi,-\psi) \leq c A \ell^{-n}\|\psi\|_{\mathrm{L}^{\infty}}$. Par conséquent, on a $\left|I_{n}(\varphi, \psi)\right| \leq c A \ell^{-n}\|\psi\|_{\mathrm{L}^{\infty}}$.

Pour le cas général, on peut supposer que $\varphi$ est à support compact dans $V$. Elle s'écrit comme différence de deux fonctions p.s.h. vérifiant les conditions ci-dessus. La constante $A$ est de l'ordre de $\|\varphi\|_{\mathcal{C}^{2}}$. On est ramené au cas précédent. 


\section{3. Équidistribution des préimages}

Dans ce paragraphe, nous étudions la distribution des préimages de $F$. Pour tout point $z \in \mathbb{C}^{k}$ et tout $n \geq 0$, posons

$$
\mu_{n}^{z}:=d_{2}^{-n}\left(F^{n}\right)^{*} \delta_{z}
$$

Notons $\mathcal{E}$ l'ensemble des points $z$ tels que la suite de mesures $\left(\mu_{n}^{z}\right)$ ne tend pas vers $\mu$. C'est l'ensemble exceptionnel de $F$. Nous avons la proposition suivante.

Proposition 3.1. - Soit F une correspondance comme au théorème 2.1. Alors $\mathcal{E}$ est pluripolaire.

Observons que puisque la mesure $\mu$ est $\mathrm{PB}$, elle ne charge pas les ensembles pluripolaires. La proposition entraîne que $\mu(\mathcal{E})=0$ et $\mu_{n}^{z}$ tend faiblement vers $\mu$ pour $\mu$-presque tout point $z \in \mathbb{C}^{k}$. On montrera plus loin que $\mathcal{E}$ est une réunion finie ou dénombrable d'ensembles algébriques.

Démonstration. - Considérons la fonction p.s.h. $\varphi:=\log \left(1+\|z\|^{2}\right)$. C'est une fonction strictement p.s.h. Posons

$$
c_{\varphi}:=\int \varphi \mathrm{d} \mu \quad \text { et } \quad \Phi:=\sum_{n=0}^{\infty}\left(\Lambda^{n} \varphi-c_{\varphi}\right)=\sum_{n=0}^{\infty}\left(\varphi_{n}-c_{\varphi}\right) .
$$

On peut appliquer la proposition 2.7 et le corollaire 2.8 à une boule $V$ arbitrairement grande. On déduit que $\Phi$ est une fonction p.s.h. sur $\mathbb{C}^{k}$. Notons $\mathcal{E}^{*}$ l'ensemble où $\Phi$ prend la valeur $-\infty$. C'est un ensemble pluripolaire. Il suffit de montrer que $\mathcal{E} \subset \mathcal{E}^{*}$.

Soit $z \notin \mathcal{E}^{*}$. Du fait que $\Phi(z)$ est finie, la suite $\varphi_{n}(z)$ tend vers $c_{\varphi}$. Soit $\psi$ une fonction réelle $\mathcal{C}^{2}$ à support compact. Pour montrer que $\mu_{n}^{z}$ tend vers $\mu$, il suffit de montrer que $\psi_{n}(z)$ tend vers $c_{\psi}:=\int \psi \mathrm{d} \mu$. Fixons $\varepsilon>0$ suffisamment petit tel que $\varphi^{ \pm}:=\varphi \pm \varepsilon \psi$ soit p.s.h. Un tel $\varepsilon$ existe car $\varphi$ est strictement p.s.h. D'après la proposition 2.7, les suites de fonctions $\varphi_{n}^{ \pm}:=\Lambda^{n} \varphi^{ \pm}$convergent vers les constantes $c^{ \pm}:=\int \varphi^{ \pm} \mathrm{d} \mu=c_{\varphi} \pm \varepsilon c_{\psi}$ dans $\mathrm{L}_{\text {loc }}^{2}\left(\mathbb{C}^{k}\right)$. Comme on l'a déjà vu dans la preuve du lemme $2.3, \lim \sup \varphi_{n}^{ \pm}(z)$ est au plus égal à $c^{ \pm}$. Or $\lim \varphi_{n}(z)=c_{\varphi}$ car $z \notin \mathcal{E}^{*}$. On en déduit que limsup $\pm \psi_{n}(z) \leq \pm c_{\psi}$, donc $\lim \psi_{n}(z)=c_{\psi}$.

Dans la suite, nous allons décrire plus précisément la distribution des préimages de $F$. Nous allons en fait construire des branches inverses holomorphes définies sur des disques et des boules.

Rappelons que $Y=\sum Y_{i}^{*}$ est le graphe de $F$. Soit $K_{0}$ un sous-ensemble connexe de $\mathbb{C}^{k}$.

On appelle branche inverse régulière d'ordre $n$ de $K_{0}$ (voir la figure 1 ) toute suite $\mathcal{B}$

$$
K_{-n},\left(\widehat{K}_{-n}, i_{n}\right), K_{-n+1},\left(\widehat{K}_{-n+1}, i_{n-1}\right), \ldots, K_{-1},\left(\widehat{K}_{-1}, i_{1}\right), K_{0}
$$

TOME $133-2005-\mathrm{N}^{\mathrm{O}} 3$ 
vérifiant les propriétés suivantes

(i) Les ensembles $K_{-m} \subset \mathbb{C}^{k}$ et $\widehat{K}_{-m} \subset Y_{i_{m}}^{*}$ sont connexes;

(ii) $\pi_{1}$ définit une bijection de $\widehat{K}_{-m}$ dans $K_{-m}$ et $\pi_{2}$ définit une bijection de $\widehat{K}_{-m}$ dans $K_{-m+1}$ pour tout $1 \leq m \leq n$.

Si $K_{0}$ n'est pas un ouvert (par exemple si $K_{0}$ est un point), on exige que les applications ci-dessus définissent des bijections holomorphes entre un voisinage de $\widehat{K}_{-m}$ et ses images. Dans ce cas, on associe $\mathcal{B}$ à ces germes de bijection holomorphe. Puisque les $Y_{i}^{*}$ ne sont pas nécessairement distincts, les indices $i_{m}$ permettent de compter les branches inverses régulières avec multiplicité. Il y a au plus $d_{2}^{n}$ branches inverses régulières d'ordre $n$ de $K_{0}$. En pratique, $K_{0}$ sera un point, un disque holomorphe, une famille de disques centrés en un point ou une boule holomorphe. Notons $F_{\mathcal{B}}^{-m}$ l'application

$$
\left(\pi_{1 \mid \widehat{K}_{-m}}\right) \circ\left(\pi_{2 \mid \widehat{K}_{-m}}\right)^{-1} \circ \cdots \circ\left(\pi_{1 \mid \widehat{K}_{-1}}\right) \circ\left(\pi_{2 \mid \widehat{K}_{-1}}\right)^{-1}
$$

pour $1 \leq m \leq n$. C'est une application holomorphe bijective de $K_{0}$ dans $K_{-m}$.

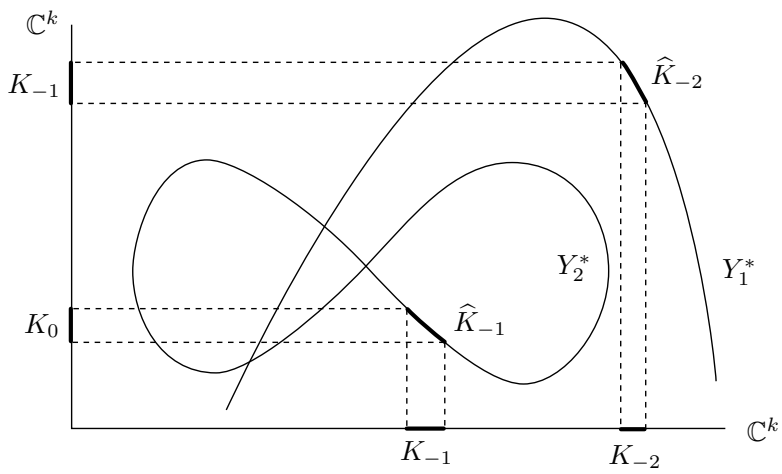

FiguRE 1. Branche inverse régulière.

Soit $\left(a_{m}\right)$ une suite de nombres réels tendant vers 0 . On dira que $\mathcal{B}$ est de taille $\left(a_{m}\right)$ si le diamètre de l'ensemble $K_{-m}$ est au plus égal à $a_{m}$ pour $0 \leq m \leq n$.

Soient $z_{0} \in K_{0}$ et $\mathcal{B}_{0}$ une branche inverse régulière d'ordre $n$ du point $z_{0}$ donnée par la suite

$$
z_{-n},\left(\widehat{z}_{-n}, i_{n}\right), z_{-n+1},\left(\widehat{z}_{-n+1}, i_{n-1}\right), \ldots, z_{-1},\left(\widehat{z}_{-1}, i_{1}\right), z_{0}
$$

(les points $\widehat{z}_{-m}$ appartiennent à $Y_{i_{m}}^{*}$ et sont de coordonnées $\left(z_{-m+1}, z_{-m}\right)$ ).

On dira que la branche $\mathcal{B}$ est accrochée à la branche $\mathcal{B}_{0}$ si on a $\widehat{z}_{-m} \in \widehat{K}_{-m}$ pour tout $1 \leq m \leq n$ (les indices $i_{n}$ dans $\mathcal{B}_{0}$ et $\mathcal{B}$ sont identiques). Pour être exact, il faut aussi supposer que les $\pi_{i \mid \widehat{K}-m}$ sont compatibles avec les germes 
de bijection holomorphes associés à $\mathcal{B}_{0}$. Ceci est nécessaire au cas où $\hat{z}-m$ est un point singulier de $Y_{i_{m}}^{*}$.

Nous soulignons ici que deux branches inverses régulières $\mathcal{B}$ et $\mathcal{B}^{\prime}$ de $K_{0}$ sont considérées comme égales si on a $K_{-m}=K_{-m}^{\prime}, \widehat{K}_{-m}=\widehat{K}_{-m}^{\prime}$ ainsi que $i_{m}=i_{m}^{\prime}$ pour tout $m$ où $K_{-m}^{\prime}, \widehat{K}_{-m}^{\prime}$ et $i_{m}^{\prime}$ sont des ensembles et des indices associés à $\mathcal{B}^{\prime}$.

Nous donnerons plus loin une notion de branche inverse plus souple qui permet de prouver que $\mathcal{E}$ est une réunion d'ensembles algébriques. Pour les branches régulières, nous avons la proposition suivante dont la preuve peut être utile dans d'autres contextes comme l'étude de la dimension de $\mu$ par exemple.

Proposition 3.2. - Soit F une correspondance comme au théorème 2.1. Alors il existe un ensemble pluripolaire $\mathcal{E}^{\prime}$ tel que pour tout $z \notin \mathcal{E}^{\prime}$, tout $\varepsilon>0$ et tout $\delta>0$, la boule $\mathrm{B}(z, r)$ possède au moins $(1-\varepsilon) d_{2}^{n}$ branches inverses régulières d'ordre $n$, de taille $\left(\ell^{-(1-\delta) m / 2}\right)$ où $r=r(z, \delta, \varepsilon)>0$ est une constante indépendante de $n$.

L'idée de la démonstration consiste à construire les branches inverses régulières pour des unions de disques holomorphes centrées en un point générique $z$. Ensuite, utilisant un théorème d'analyse complexe (lemme 3.8), on peut prolonger les applications holomorphes $F_{\mathcal{B}}^{-n}$ associées à ces branches inverses régulières. Ces applications sont définies aux voisinages de grandes familles de disques centrés en $z$. On les prolonge en applications holomorphes sur une petite boule $\mathrm{B}(z, r)$. Ces prolongements fournissent les branches inverses régulières pour $\mathrm{B}(z, r)$.

La construction est faite par récurrence. Nous résumons ici le passage du rang $n-1$ au rang $n$. Soit $K_{-n+1}$ un disque holomorphe fabriqué au rang $n-1$ à partir d'un disque $K_{0}$ de rayon $r^{\prime}>0$ centré en $z$. Nous voulons en fait construire des branches inverses régulières d'ordre 1 de $K_{-n+1}$. D'abord, pour construire $\widehat{K}_{-n}$, on veut que $K_{-n+1}$ ne rencontre pas les valeurs critiques de $\pi_{2 \mid Y}$; ensuite, pour obtenir $K_{-n}$, nous avons besoin que $\pi_{1 \mid \widehat{K}_{-n}}$ soit injective.

Pour que ces deux conditions soient satisfaites, nous sélectionnons seulement les disques $K_{-n+1}$ de diamètre assez petit qui ne sont pas trop proches d'un certain sous-ensemble algébrique $(P=0)$ que nous appelons l'ensemble des valeurs critiques de $F$. Pour chaque disque sélectionné, on peut construire $d_{2}$ branches inverses régulières d'ordre 1 . Afin de continuer la construction, nous devons sélectionner, parmi les nouveaux disques, ceux de petit diamètre qui ne sont pas trop proches de $(P=0)$. L'hypothèse sur l'exposant de Lojasiewicz implique qu'un bon nombre de disques sont de petite aire. Ces disques ne sont pas forcément de petit diamètre. Mais, en diminuant légèrement $r^{\prime}$, on rend leur diamètre petit. Le fait que $\mu$ soit une mesure PB implique que pour $z$ générique, il n'y a qu'un petit nombre de disques qui sont proches de $(P=0)$.

TOME $133-2005-\mathrm{N}^{\mathrm{O}} 3$ 
Soit $Q$ un polynôme non nul. Pour tout $\alpha>0$, notons $\mathcal{V}_{Q}(\alpha)$ l'ensemble des points $z$ vérifiant $|Q(z)| \leq \alpha$. Le lemme suivant montre que les préimages de $F$ ne sont pas «trop proches » de l'hypersurface $(Q=0)$. On peut le généraliser aux ensembles pluripolaires de $\mathbb{P}^{k}$.

Lemme 3.3. - Soit $a>1$ une constante. Alors il existe un ensemble pluripolaire $\mathcal{E}_{Q}^{a}$ tel que $\sum_{n \geq 0} \mu_{n}^{z}\left(\mathcal{V}_{Q}\left(a^{-n}\right)\right)$ soit fini pour tout $z \notin \mathcal{E}_{Q}^{a}$.

Démonstration. - On peut supposer $a<\ell^{1 / 2}$. On choisit une fonction $\chi$ lisse, positive sur $\mathbb{R}^{+}$telle que $\chi \leq 1, \chi=1$ sur $[0,1]$ et $\chi=0$ sur $[2,+\infty[$. Soit $\tau \leq 1$ une fonction lisse, positive à support dans $V$ et égale à 1 au voisinage de $\bar{U}$. Posons $\psi^{n}:=\tau \chi\left(a^{n} Q\right)$. Les mesures $\mu_{n}^{z}$ étant portées par $\bar{U}$ pour $n$ assez grand, il suffit de montrer que la somme $\sum_{n \geq 0}\left\langle\mu_{n}^{z}, \psi^{n}\right\rangle$ est finie pour tout $z \notin \mathcal{E}_{Q}^{a}$ où l'ensemble $\mathcal{E}_{Q}^{a}$ sera précisé dans la suite.

D'après la proposition 2.7, la fonction $\log |Q|$ est $\mu$-intégrable. On en déduit que la somme $\sum_{n \geq 0}\left\langle\mu, \psi^{n}\right\rangle$ est finie. En effet, on a

$$
\begin{aligned}
\sum_{n \geq 0}\left\langle\mu, \psi^{n}\right\rangle & \leq \sum_{n \geq 0} \mu\left(\mathcal{V}_{Q}\left(2 a^{-n}\right)\right)=\int\left(\sum_{n \geq 0} \mathbf{1}_{\mathcal{V}_{Q}\left(2 a^{-n}\right)}\right) \mathrm{d} \mu \\
& \leq \int \frac{1}{\log a}(|\log | Q||+\log 2+\log a) \mathrm{d} \mu .
\end{aligned}
$$

Posons $c_{n}:=\left\langle\mu, \psi^{n}\right\rangle$. Par définition de $\psi^{n}$, il existe une constante $c>0$ telle que $\left\|\psi^{n}\right\|_{\mathcal{C}^{2}} \leq c a^{2 n}$. Posons $\varphi:=\log \left(1+\|z\|^{2}\right)$ et $\psi^{n-}:=c a^{2 n} \varphi-\psi^{n}$. Ce sont des fonctions p.s.h. à croissance logarithmique. Posons

$$
c_{\varphi}:=\langle\mu, \varphi\rangle \quad \text { et } \quad c_{n}^{-}:=\left\langle\mu, \psi^{n-}\right\rangle .
$$

On a

$$
c_{n}=c a^{2 n} c_{\varphi}-c_{n}^{-} .
$$

Rappelons qu'on a supposé $a<\ell^{1 / 2}$. D'après la proposition 2.7 et le corollaire 2.8 , la série de fonctions

$$
\Phi^{+}:=\sum_{n \geq 0} c a^{2 n}\left(\Lambda^{n} \varphi-c_{\varphi}\right)
$$

converge ponctuellement vers une fonction p.s.h. En particulier, $\Phi^{+}$est localement bornée supérieurement. La proposition 2.7 et le corollaire 2.8 appliqués aux fonctions $\psi^{n-}$ impliquent aussi que la série de fonctions

$$
\Phi^{-}:=\sum_{n \geq 0}\left(\Lambda^{n} \psi^{n-}-c_{n}^{-}\right)
$$

converge ponctuellement vers une fonction p.s.h.

BULLETIN DE LA SOCIÉtÉ MATHÉMATIQUE DE FRANCE 
Notons $\mathcal{E}_{Q}^{a}$ l'ensemble où $\Phi^{-}$vaut $-\infty$. C'est un ensemble pluripolaire. Pour $z \notin \mathcal{E}_{Q}^{a}$, on a

$$
\begin{aligned}
\sum_{n \geq 0}\left\langle\mu_{n}^{z}, \psi^{n}\right\rangle-\sum_{n \geq 0}\left\langle\mu, \psi^{n}\right\rangle & =\sum_{n \geq 0}\left(\left\langle\mu_{n}^{z}, \psi^{n}\right\rangle-c_{n}\right) \\
& =\sum_{n \geq 0} c a^{2 n}\left(\left\langle\mu_{n}^{z}, \varphi\right\rangle-c_{\varphi}\right)-\sum_{n \geq 0}\left(\left\langle\mu_{n}^{z}, \psi^{n-}\right\rangle-c_{n}^{-}\right) \\
& =\Phi^{+}(z)-\Phi^{-}(z)<+\infty
\end{aligned}
$$

Or la somme $\sum\left\langle\mu, \psi^{n}\right\rangle$ est finie comme on l'a montré ci-dessus. La somme $\sum\left\langle\mu_{n}^{z}, \psi^{n}\right\rangle$ est donc aussi finie.

LEMME 3.4. - Il existe un polynôme $P$ (indépendant de $U, V$ ) et une constante $A_{1}>0$ (dépendante de $\left.U, V\right)$ tels que pour tout point $z \in V \backslash(P=0)$ la boule $\mathrm{B}_{0}:=\mathrm{B}\left(z, r_{0}\right)$ admette exactement $d_{t}$ branches inverses régulières d'ordre 1 pour $F$, où $r_{0}:=A_{1}|P(z)|$.

Démonstration. - Notons $Z:=|Y|$ le support de $Y$ et $m_{2}$ le degré de l'application $\pi_{2 \mid Z}$. Soit $\Sigma_{2}$ l'ensemble des points $z \in \mathbb{C}^{k}$ tels que $\left(\pi_{2 \mid Z}\right)^{-1}(z)$ contienne moins de $m_{2}$ points. C'est un sous-ensemble algébrique de $\mathbb{C}^{k}$. Il existe donc un polynôme $P_{1}$ tel que $\Sigma_{2} \subset\left(P_{1}=0\right)$. Définissons de la même manière l'ensemble des valeurs critiques $\Sigma_{1}$ de $\pi_{1 \mid Z}$ et posons $\Sigma^{\prime}:=F\left(\Sigma_{1}\right)$. Soit $P_{2}$ un polynôme tel que $\Sigma^{\prime} \subset\left(P_{2}=0\right)$. Posons $P=P_{1}^{m} P_{2}^{m}$ où $m \geq 1$ est un entier assez grand.

Soit $A_{1}>0$ une constante assez petite. Puisque $A_{1}$ est petite et $m$ est grand, $\left(\pi_{2 \mid Z}\right)^{-1} \mathrm{~B}_{0}$ contient exactement $m_{2}$ composantes connexes et la restriction de $\pi_{1}$ à chacune de ces composante est injective.

Soit $\widehat{\mathrm{B}}_{-1}$ une composante connexe de $\pi_{2}^{-1}\left(\mathrm{~B}_{0}\right) \cap Y_{i}^{*}$, pour un certain indice $i$. La suite $\mathrm{B}_{-1},\left(\widehat{\mathrm{B}}_{-1}, i_{1}\right), \mathrm{B}_{0}$ avec $i_{1}:=i$ et $\mathrm{B}_{-1}:=\pi_{1}\left(\widehat{\mathrm{B}}_{-1}\right)$, est une branche inverse régulière d'ordre 1 de $\mathrm{B}_{0}$. Il y a exactement $d_{2}$ branches.

Notons $\mathcal{E}_{1}$ l'orbite de $(P=0)$ par $\left(F^{n}\right)_{n \geq 0}$. Par définition, tout point $z \notin \mathcal{E}_{1}$ admet $d_{2}^{n}$ branches inverses d'ordre $n$. Posons $\mathcal{E}^{\prime}:=\mathcal{E}_{P}^{a} \cup \mathcal{E}_{1}$. Il est clair que $\mathcal{E}^{\prime}$ est pluripolaire.

Fixons maintenant des constantes $\delta$ avec $0<\delta<1$, a avec $1<a<\ell^{(1-\delta) / 2}$, $\varepsilon$ avec $0<\varepsilon<1$ et un point $z \in V \backslash \mathcal{E}^{\prime}$. Nous allons montrer que la boule $\mathrm{B}(z, r)$ possède au moins $(1-\varepsilon) d_{2}^{n}$ branches inverses régulières d'ordre $n$ et de taille $\left(\ell^{-(1-\delta) m / 2}\right)$ pour $r>0$ assez petit.

Fixons une droite $\Delta$ passant par $z$. Notons $\Delta_{R}$ le disque de centre $z$ et de rayon $R$ dans $\Delta$. Rappelons le lemme de comparaison aire-diamètre dû̀ à Briend-Duval [4]. Ce lemme est valable pour un cas plus général. Dans le cas présent, on peut le montrer en utilisant la formule de Cauchy.

LEMME 3.5. - Soient $\pi$ une application holomorphe du disque unité $\mathrm{D}:=\mathrm{D}(0,1)$ dans $U$ et $\tau$ une constante vérifiant $0<\tau<1$. Alors il 
existe une constante $A_{2}>0$ indépendante de $\pi$ et de $\tau$ telle que le diamètre de $\pi(\mathrm{D}(0,1-\tau))$ soit plus petit ou égal à $A_{2} \sqrt{\tau^{-1} \text { aire }(\pi(\mathrm{D}))}$, les points de $\pi(\mathrm{D})$ étant comptés avec multiplicité.

Nous allons montrer la proposition suivante dans laquelle la constante $A_{3}>1$ sera donnée dans le lemme 3.8.

Proposition 3.6. - Pour tout $\varepsilon_{1}>0$, il existe $r>0$ et $n_{0} \geq 0$ indépendants de $\Delta$ tels que $\Delta_{A_{3} r}$ possède au moins $\left(1-\varepsilon_{1}\right) d_{t}^{n}$ branches inverses régulières $\mathcal{B}$ d'ordre $n$, de taille $\left(\frac{1}{2} \ell^{-(1-\delta) m / 2}\right)$ et telles que $\left|P\left(F_{\mathcal{B}}^{-m}(z)\right)\right| \geq a^{-m}$ pour tout $n_{0} \leq m \leq n$.

Fixons $n_{0} \geq 1$ assez grand tel que les propriétés suivantes soient satisfaites :

1) $A_{1} a^{-n}>\ell^{-(1-\delta) n / 2}$ pour tout $n \geq n_{0}$,

2) $\sum_{n \geq n_{0}+1} \mu_{n}^{z}\left(\mathcal{V}_{P}\left(a^{-n}\right)\right) \leq \frac{1}{2} \varepsilon_{1}$,

3) $\sum_{n \geq n_{0}+1} n^{2} \ell^{-\delta n} \leq \frac{1}{8} \varepsilon_{1} A_{2}^{-2}$.

Posons

$$
\nu_{m}:=\sum_{n=n_{0}+1}^{m} \mu_{n}^{z}\left(\mathcal{V}_{P}\left(a^{-n}\right)\right), \quad \delta_{m}:=4 A_{2}^{2} \sum_{n=n_{0}+1}^{m} n^{2} \ell^{-\delta n} .
$$

Fixons $r_{1}>0$ assez petit tel que pour tout $n \leq n_{0}, \mathrm{~B}\left(z, r_{1}\right)$ possède exactement $d_{2}^{n}$ branches inverses régulières d'ordre $n$ de taille $\left(\frac{1}{2} \ell^{-(1-\delta) m / 2}\right)$. Posons pour tout $n \geq n_{0}+1$

$$
r_{n}:=\prod_{s=n_{0}+1}^{n}\left(1-\frac{1}{s^{2}}\right) r_{1}
$$

Cette suite $\left(r_{n}\right)$ décroît vers une constante $A_{3} r>0$. Il suffit pour la proposition 3.6 de montrer par récurrence sur $n \geq n_{0}$ que $\Delta_{r_{n}}$ possède au moins $\left(1-\nu_{n}-\delta_{n}\right) d_{2}^{n}$ branches inverses régulières $\mathcal{B}$ d'ordre $n$, de taille $\left(\frac{1}{2} \ell^{-(1-\delta) m / 2}\right)$ et telles que $\left|P\left(F_{\mathcal{B}}^{-m}(z)\right)\right| \geq a^{-m}$ pour tout $n_{0} \leq m \leq n$. Supposons que c'est vrai au rang $n-1 \geq n_{0}$. Montrons-le au rang $n$. Notons $\mathcal{F}$ la famille des branches inverses régulières d'ordre $n-1$ de $\Delta_{r_{n-1}}$ vérifiant la proposition 3.6.

Si $\mathcal{B}$ est un élément de $\mathcal{F}$, d'une part, le point $w:=F_{\mathcal{B}}^{-n+1}(z)$ vérifie $|P(w)| \geq a^{-n+1}$, d'autre part, l'ensemble $W:=F_{\mathcal{B}}^{-n+1}\left(\Delta_{r_{n-1}}\right)$ est contenu dans la boule $\mathrm{B}\left(w, A_{1} a^{-n+1}\right)$ car son diamètre est plus petit que $\frac{1}{2} A_{1} a^{-n+1}$. Or, d'après le lemme 3.4 , cette boule $\mathrm{B}\left(w, A_{1} a^{-n+1}\right)$ admet exactement $d_{2}$ branches inverses régulières d'ordre 1 . On en déduit que $W$ admet aussi $d_{2}$ branches inverses régulières d'ordre 1 . Ceci est vrai pour tout $\mathcal{B} \in \mathcal{F}$. En somme, $\Delta_{r_{n-1}}$ possède au moins $\left(1-\nu_{n-1}-\delta_{n-1}\right) d_{2}^{n}$ branches inverses régulières d'ordre $n$. Notons $\mathcal{G}$ cette famille de branches inverses. On a le lemme suivant.

LEMME 3.7. - La somme $\sum \operatorname{aire}\left(F_{\mathcal{B}}^{-n}\left(\Delta_{r_{n-1}}\right)\right)$ pour $\mathcal{B} \in \mathcal{G}$ est plus petite que $d_{2}^{n} \ell^{-n}$. 
Démonstration. - Notons $[\Delta]$ le courant d'intégration sur $\Delta$. Il suffit de majorer la masse de $\left(F^{n}\right)^{*}[\Delta]$ par $d_{2}^{n} \ell^{-n}$. On a d'après un lemme de comparaison (voir [34] ou [14, prop. 5.4])

$$
\begin{aligned}
\left\|\left(F^{n}\right)^{*}[\Delta]\right\| & =\left\langle\left(F^{n}\right)^{*}[\Delta], \omega\right\rangle=\left\langle[\Delta],\left(F^{n}\right)_{*} \omega\right\rangle \\
& =\frac{1}{2} d_{2}^{n} \ell^{-n} \int_{\Delta} \operatorname{dd}^{\mathrm{c}}\left(\ell^{n} \Lambda^{n} \log \left(1+\|z\|^{2}\right)\right) \\
& \leq \frac{1}{2} d_{2}^{n} \ell^{-n} \int_{\Delta} \operatorname{dd}^{\mathrm{c}} \log \left(1+\|z\|^{2}\right)=d_{2}^{n} \ell^{-n} \int_{\Delta} \omega=d_{2}^{n} \ell^{-n} .
\end{aligned}
$$

L'inégalité ci-dessus est une conséquence du fait que

$$
\ell^{n} \Lambda^{n} \log \left(1+\|z\|^{2}\right)-\log \left(1+\|z\|^{2}\right)
$$

est bornée supérieurement.

Fin de la démonstration de la proposition 3.6. - Notons $\mathcal{G}_{1}$ l'ensemble des branches inverses régulières $\mathcal{B} \in \mathcal{G}$ telles que l'aire de $F_{\mathcal{B}}^{-n}\left(\Delta_{r_{n-1}}\right)$ excède $\frac{1}{4} A_{2}^{-2} n^{-2} \ell^{-(1-\delta) n}$. Alors $\mathcal{G}_{1}$ contient au plus $4 A_{2}^{2} n^{2} \ell^{-\delta n} d_{2}^{n}$ éléments d'après le lemme 3.7. Par conséquent, le cardinal de la famille $\mathcal{G}^{\prime}:=\mathcal{G} \backslash \mathcal{G}_{1}$ est au moins égal à

$$
\# \mathcal{G}-\# \mathcal{G}_{1} \geq\left(1-\nu_{n-1}-\delta_{n-1}\right) d_{2}^{n}-4 A_{2}^{2} n^{2} \ell^{-\delta n} d_{2}^{n}=\left(1-\nu_{n-1}-\delta_{n}\right) d_{2}^{n} .
$$

D'après le lemme 3.5 (appliqué à $F_{\mathcal{B}}^{-n}$ sur les disques $\Delta_{r_{n-1}}$ et $\Delta_{r_{n}}$ ), le diamètre de $F_{\mathcal{B}}^{-n}\left(\Delta_{r_{n}}\right)$ est au plus égal à $\frac{1}{2} \ell^{-(1-\delta) n / 2}$ pour tout $\mathcal{B} \in \mathcal{G}^{\prime}$.

Notons $\mathcal{G}_{2}$ la famille des éléments $\mathcal{B} \in \mathcal{G}^{\prime}$ tels que $F_{\mathcal{B}}^{-n}(z) \in \mathcal{V}_{P}\left(a^{-n}\right)$. Alors $\mathcal{G}_{2}$ contient au plus $\mu_{n}^{z}\left(\mathcal{V}_{P}\left(a^{-n}\right)\right) d_{2}^{n}$ éléments. Par conséquent, la famille $\mathcal{G}^{\prime \prime}:=$ $\mathcal{G}^{\prime} \backslash \mathcal{G}_{2}$ contient au moins

$$
\left(1-\nu_{n-1}-\delta_{n}\right) d_{2}^{n}-\mu_{n}^{z}\left(\mathcal{V}_{P}\left(a^{-n}\right)\right) d_{2}^{n}=\left(1-\nu_{n}-\delta_{n}\right) d_{2}^{n}
$$

éléments. Cette famille $\mathcal{G}^{\prime \prime}$ de branches inverses régulières d'ordre $n$ de $F$ vérifie la proposition 3.6.

Fin de la démonstration de la proposition 3.2. - Prenons $\varepsilon_{1}=\frac{1}{2} \varepsilon$ et notons $\mathcal{W}$ la famille des droites complexes passant par $z$. Cette famille est paramétrée par l'espace projectif $\mathbb{P}^{k-1}$. Notons $\mathcal{H}_{2 k-2}$ la mesure de Hausdorff $(2 k-2)$ dimensionnelle de masse 1 sur $\mathcal{W}$. Notons également $\mathcal{F}_{n}$ la famille des branches inverses régulières d'ordre $n$ de $z$. Cette famille contient exactement $d_{2}^{n}$ éléments car $z \notin \mathcal{E}_{1}$. Pour tout $\mathcal{B}_{z} \in \mathcal{F}_{n}$ notons $\mathcal{W}_{\mathcal{B}_{z}}$ la famille des droites $\Delta \in \mathcal{W}$ telles que $\Delta_{A_{3} r}$ possède une branche inverse régulière d'ordre $n$, accrochée à la branche $\mathcal{B}_{z}$ et vérifiant les propriétés dans la proposition 3.6. D'après cette proposition, on a $\sum_{\mathcal{B}_{z}} \mathcal{H}_{2 k-2}\left(\mathcal{W}_{\mathcal{B}_{z}}\right) \geq\left(1-\varepsilon_{1}\right) d_{2}^{n}$ lorsque $r>0$ est suffisamment petit. Notons $\mathcal{F}_{n}^{\prime}$ la famille des $\mathcal{B}_{z}$ tels que $\mathcal{H}_{2 k-2}\left(\mathcal{W}_{\mathcal{B}_{z}}\right) \geq \frac{1}{2}$. Du fait que $\mathcal{H}_{2 k-2}\left(\mathcal{W}_{\mathcal{B}_{z}}\right) \leq 1$ pour tout $\mathcal{B}_{z}$, on a

$$
\# \mathcal{F}_{n}^{\prime}+\frac{1}{2}\left(d_{2}^{n}-\# \mathcal{F}_{n}^{\prime}\right) \geq \sum \mathcal{H}_{2 k-2}\left(\mathcal{W}_{\mathcal{B}_{z}}\right) \geq\left(1-\varepsilon_{1}\right) d_{2}^{n}
$$

TOME $133-2005-\mathrm{N}^{\mathrm{O}} 3$ 
On en déduit que $\# \mathcal{F}_{n}^{\prime} \geq\left(1-2 \varepsilon_{1}\right) d_{2}^{n}=(1-\varepsilon) d_{2}^{n}$. On va appliquer le théorème de Sibony-Wong suivant pour chaque $\mathcal{W}_{\mathcal{B}_{z}}$ avec $\mathcal{B}_{z} \in \mathcal{F}_{n}^{\prime}$.

Lemme 3.8 (voir [1], [35]). - Soit $\alpha>0$ une constante positive. Soit $A_{3}>1$ une constante suffisamment grande et soit $\mathcal{W}^{\prime}$ une famille de droites passant par z. Supposons que $\mathcal{H}_{2 k-2}\left(\mathcal{W}^{\prime}\right) \geq \alpha$. Notons $\Sigma$ l'intersection de ces droites avec la boule $\mathrm{B}\left(z, A_{3} r\right)$. Alors toute application holomorphe $f$ d'un voisinage de $\Sigma$ d'image dans $\mathbb{C}^{k}$ se prolonge en application holomorphe de $\mathrm{B}(z, r)$ dans $\mathbb{C}^{k}$. De plus, on a

$$
\sup _{w \in \mathrm{B}(z, r)}\|f(w)-f(z)\| \leq \sup _{w \in \Sigma}\|f(w)-f(z)\| .
$$

En particulier, on a $\operatorname{diam} f(\mathrm{~B}(z, r)) \leq 2 \operatorname{diam} f(\Sigma)$.

On prend $\alpha=\frac{1}{2}$. Fixons $\mathcal{B}_{z} \in \mathcal{F}_{n}^{\prime}$. Notons $K_{0}$ l'intersection des droites de $\mathcal{W}_{\mathcal{B}_{z}}$ avec $\mathrm{B}\left(z, A_{3} r\right)$ et $\mathcal{B}$ sa branche inverse régulière d'ordre $n$ accrochée à $\mathcal{B}_{z}$. D'après le lemme 3.8 , l'application $F_{\mathcal{B}}^{-m}$, qui est holomorphe au voisinage de $K_{0}$, se prolonge en une application holomorphe de $\mathrm{B}(z, r)$ dans $\mathbb{C}^{k}$ pour tout $1 \leq m \leq n$. De plus, son image est de diamètre au plus $\ell^{-(1-\delta) m / 2}$. Les applications obtenues sont injectives. En effet, on montre par récurrence que l'image de $\mathrm{B}(z, r)$ par $F_{\mathcal{B}}^{-m}$ est contenue dans la boule de rayon $A_{1}\left|P\left(F_{\mathcal{B}}^{-m}(z)\right)\right|$ centrée en $F_{\mathcal{B}}^{-m}(z)$ (voir le lemme 3.4). Chaque prolongement holomorphe fournit une branche inverse régulière d'ordre $n$, de taille $\left(\ell^{-(1-\delta) m / 2}\right)$ pour la boule $\mathrm{B}(z, r)$. Ceci termine la preuve de la proposition 3.2.

La suite de ce paragraphe a été démontrée en collaboration avec Charles Favre.

Notons $\mathrm{PC}_{1}$ l'ensemble des points $z \in \mathbb{C}^{k}$ tels que au moins un des germes locaux irréductibles de $Y$ en $\pi_{2}^{-1}(z) \cap Y$ ne se projette pas injectivement par $\pi_{2}$ sur $\mathbb{C}^{k}$. C'est une hypersurface de $\mathbb{C}^{k}$. Soit $S_{1}$ le courant d'intégration sur $\mathrm{PC}_{1}$. Posons

$$
S_{n}:=\left(F^{n-1}\right)_{*}\left(S_{1}\right), \quad S:=\sum_{n \geq 1} d_{2}^{-n+1} S_{n} .
$$

Ce sont des courants positifs fermés de bidegré $(1,1)$ de $\mathbb{C}^{k}$. Le courant $S$ est bien défini car d'après le lemme 2.2, la masse de $d_{2}^{-n} S_{n}$ est de l'ordre de $\ell^{-n}$. Soient $\mathrm{PC}_{n}:=\bigcup_{i=1}^{n} \operatorname{supp}\left(S_{i}\right)$ et $\mathrm{PC}_{\infty}:=\operatorname{supp}(S)$ les ensembles postcritiques d'ordre $n$ et d'ordre infini de $F$. Posons également $\Sigma:=Y \cap \pi_{2}^{-1}\left(\mathrm{PC}_{1}\right)$.

Soit $K_{0}$ un disque holomorphe de centre $z$, une famille de disques holomorphes centrés en $z$ ou une boule de centre $z$. On ne considère que les disques holomorphes plats qui ne sont pas contenus dans $\mathrm{PC}_{\infty}$. On appelle branche inverse d'ordre $n$ de $K_{0}$ (voir la figure 2 ) toute suite $\mathcal{B}$

$$
K_{-n},\left(\widehat{K}_{-n}, i_{n}\right), K_{-n+1}, \ldots, K_{-1},\left(\widehat{K}_{-1}, i_{1}\right), K_{0}
$$

munie des applications holomorphes $\widehat{F}_{\mathcal{B}}^{-m}: K_{0} \longrightarrow \widehat{K}_{-m}$ telle que 
(i) les ensembles $K_{-m} \subset \mathbb{C}^{k}, \widehat{K}_{-m} \subset Y_{i_{m}}^{*} \backslash \Sigma$ sont connexes; $\pi_{1}\left(\widehat{K}_{-m}\right)=K_{-m}, \pi_{2}\left(\widehat{K}_{-m}\right)=K_{-m+1}$ pour $1 \leq m \leq n$;

(ii) $\pi_{2} \circ \widehat{F}_{\mathcal{B}}^{-1}=$ id et $\pi_{1} \circ \widehat{F}_{\mathcal{B}}^{-m+1}=\pi_{2} \circ \widehat{F}_{\mathcal{B}}^{-m}$ pour tout $2 \leq m \leq n$.

On n'exige pas que $\pi_{1 \mid \widehat{K}_{-n}}$ soit injective. Posons $F_{\mathcal{B}}^{-m}:=\pi_{1} \circ \widehat{F}_{\mathcal{B}}^{-m}$.

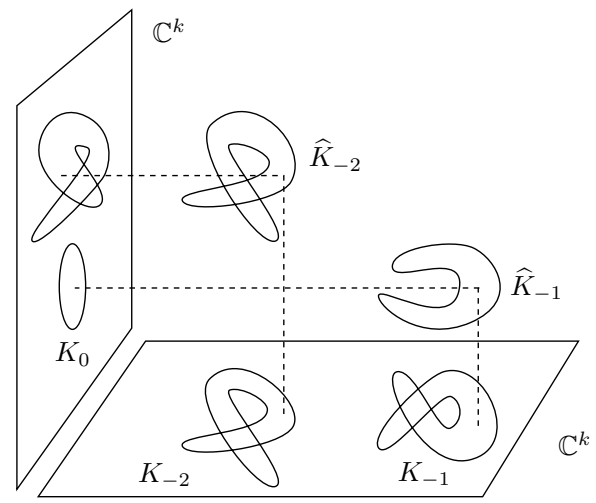

FiguRE 2. Branche inverse non régulière.

Fixons un point $w \in K_{0}$. La branche inverse $\mathcal{B}$ de $K_{0}$ est accrochée à la branche inverse régulière $\mathcal{B}_{w}$ de $w$ donnée par la suite

$$
w_{-n},\left(\widehat{w}_{-n}, i_{n}\right), w_{-n+1},\left(\widehat{w}_{-n+1}, i_{n-1}\right), \ldots, w_{-1},\left(\widehat{w}_{-1}, i_{1}\right), w_{0}
$$

où $w_{0}:=w, \widehat{w}_{-m}:=\widehat{F}_{\mathcal{B}}^{-m}\left(w_{0}\right)$ et $w_{-m}:=\pi_{1}\left(\widehat{w}_{-m}\right)$. Par unicité du prolongement analytique, la branche $\mathcal{B}$ est uniquement déterminée par la branche $\mathcal{B}_{w}$. Autrement dit, si deux branches inverses d'ordre $n$ de $K_{0}$ sont accrochées à une même branche régulière $\mathcal{B}_{w}$, alors elles sont égales. On en déduit que $K_{0}$ possède au plus $d_{2}^{n}$ branches inverses d'ordre $n$. Nous avons le théorème suivant.

THÉORÈme 3.9. - Soit F une correspondance comme au théorème 2.1. Soit $z \in \mathbb{C}^{k}$ un point tel que le nombre de Lelong de $S$ en $z$ vérifie $\nu(S, z)<1$. Alors pour tout $\varepsilon>0$ et tout $\delta>0$, la boule $\mathrm{B}(z, r)$ possède au moins $(1-\nu(S, z)-\varepsilon) d_{2}^{n}$ branches inverses d'ordre $n$ de taille $\left(\ell^{-(1-\delta) m / 2}\right)$ où $r>0$ est une constante indépendante de $n$.

Notons que ce théorème a été prouvé dans [13] pour les endomorphismes holomorphes de degré $d \geq 2$ de $\mathbb{P}^{k}$ (généralement pour les applications d'allure polynomiale de grand degré topologique). Dans ce cas, les branches inverses sont de taille $\left(\simeq d^{-m / 2}\right)$. Indépendemment, dans une note non publiée, BriendDuval ont construit les branches inverses sur les boules par une autre méthode. Ils ont montré que la taille de ces branches tend vers 0 quand $n \rightarrow \infty$. Les

TOME $133-2005-\mathrm{N}^{\mathrm{O}} 3$ 
branches inverses sur les disques ont été déjà construites dans [4]. Le lecteur trouvera dans [10] d'autre version du théorème 3.9 avec des applications.

Pour prouver ce théorème, il suffit de construire des branches inverses pour des disques holomorphes plats centrés en $z$. Les deux arguments suivants permettent d'adapter la preuve de la proposition 3.2.

1) Fixons $r^{\prime}>0$ assez petit. Notons $s_{n}$ le nombre de points d'intersection de $\Delta_{r^{\prime}}$ avec $F^{n-1}\left(\mathrm{PC}_{1}\right)$, c'est-à-dire la masse de la mesure d'intersection de $\left[\Delta_{r^{\prime}}\right]$ avec le courant $S_{n}$. Ce nombre $s_{n}$ est aussi égal à la masse de la mesure d'intersection de $S_{1}$ avec $\left(F^{n-1}\right)^{*}\left[\Delta_{r^{\prime}}\right]$. Notons $\mathcal{W}^{\prime}$ la famille des droites $\Delta$ telles que $\sum d_{2}^{-n+1} s_{n} \leq \nu(S, z)+\varepsilon$. Par tranchage, si $r^{\prime}$ est suffisamment petit, on a $\mathcal{H}_{2 k-2}\left(\mathcal{W}^{\prime}\right) \geq \frac{1}{2}$.

2) Fixons un $\Delta$ dans $\mathcal{W}^{\prime}$. On construit, par récurrence sur $n$, au moins

$$
N_{n}:=d_{2}^{n}\left(1-\sum_{m=1}^{n} d_{2}^{-m+1} s_{m}\right)
$$

branches inverses d'ordre $n$ pour $\Delta_{r^{\prime}}$. Résumons le passage du rang $n-1$ au rang $n$. Par définition de $s_{n}$, il existe au moins $N_{n-1}-s_{n}$ branches inverses $\mathcal{B}$ d'ordre $n-1$ de $\Delta_{r^{\prime}}$

$$
K_{-n+1},\left(\widehat{K}_{-n+1}, i_{n-1}\right), \ldots, K_{-1},\left(\widehat{K}_{-1}, i_{1}\right), K_{0}
$$

avec $K_{0}=\Delta_{r^{\prime}}$ telles que l'ensemble $F_{\mathcal{B}}^{-n+1}\left(\Delta_{r^{\prime}}\right)$, qui est égal à $K_{-n+1}$, ne rencontre pas $\mathrm{PC}_{1}$. D'autre part, l'application $\pi_{2}$ définit un revêtement non ramifié de degré $d_{2}$ de $Y \backslash \Sigma$ au-dessus de $\mathbb{C}^{k} \backslash \mathrm{PC}_{1}$. L'ensemble $\Delta_{r^{\prime}}$ étant simplement connexe, on peut construire, pour une telle branche $\mathcal{B}$, exactement $d_{2}$ applications holomorphes $\tau$ de $\Delta_{r^{\prime}}$ dans un des $Y_{i}^{*}$ telles que $\pi_{2} \circ \tau=F_{\mathcal{B}}^{-n+1}$. On obtient donc au moins $N_{n}$ branches inverses $\mathcal{B}^{\prime}$ d'ordre $n$ de $\Delta_{r^{\prime}}$

$$
K_{-n},\left(\widehat{K}_{-n}, i_{n}\right), \ldots, K_{-1},\left(\widehat{K}_{-1}, i_{1}\right), K_{0}
$$

en posant $i_{n}:=i, \widehat{F}_{\mathcal{B}^{\prime}}^{-n}:=\tau, \widehat{K}_{-n}:=\tau\left(\Delta_{r^{\prime}}\right)$ et $K_{-n}:=\pi_{1}\left(\widehat{K}_{-n}\right)$.

Corollaire 3.10. - Soit F une correspondance comme au théorème 2.1. Alors l'ensemble exceptionnel $\mathcal{E}$ de $F$ est égal à $\bigcup_{n \geq 0} F^{n}\left(\mathcal{E}_{0}\right)$ où $\mathcal{E}_{0}$ est le plus grand sous-ensemble algébrique propre de $\mathbb{C}^{k}$ vérifiant $F^{-1}\left(\mathcal{E}_{0}\right)=\mathcal{E}_{0}$.

Démonstration. - Pour tout $\nu>0$, notons $X_{\nu}$ l'ensemble des points $z$ tels que $\nu(S, z) \geq \nu$. C'est un sous-ensemble algébrique de $\mathbb{C}^{k}$ contenu dans $\mathrm{PC}_{\infty}$. Soient $z \notin X_{\nu}$ avec $\nu<1, \varepsilon$ et $\mathrm{B}(z, r)$ vérifiant le théorème 3.9. D'après la proposition 3.1, pour un point générique $w \in \mathrm{B}(z, r)$, on a $\lim \mu_{n}^{w}=\mu$. Considérons une valeur adhérente $\mu^{z}$ de la suite de mesures $\left(\mu_{n}^{z}\right)$ et $\mu_{\text {reg }}^{z}$ sa partie absolument continue par rapport à $\mu$. D'après le théorème $3.9, z$ et $w$ possèdent au moins $d_{2}^{n}(1-\nu-\varepsilon)$ images réciproques $z_{-n}$ et $w_{-n}$ d'ordre $n$ telles que la distance entre $z_{-n}$ et $w_{-n}$ soit majorée par $\ell^{-(1-\delta) n / 2}$. On en 
déduit que la masse de $\mu_{\text {reg }}^{z}$ est au moins égale à $1-\nu-\varepsilon$. Donc elle est au moins égale à $1-\nu$. On déduit aussi que $\mathcal{E} \subset \bigcup_{\nu>0} X_{\nu} \subset \mathrm{PC}_{\infty}$.

Notons $\mathcal{E}_{X_{\nu}}$ l'ensemble des points $z$ tels que $F^{-n}(z) \subset X_{\nu}$ pour tout $n \geq 0$. C'est le plus grand sous-ensemble algébrique de $X_{\nu}$ qui vérifie $F^{-1}\left(\mathcal{E}_{X_{\nu}}\right) \subset \overline{\mathcal{E}}_{X_{\nu}}$. Il est clair que $\mathcal{E}_{X_{\nu}} \subset \mathcal{E}$. Pour tout $z \in \mathbb{C}^{k}$, posons

$$
\widetilde{\mu}_{0}^{z}:=\mathbf{1}_{X_{\nu}} \delta_{z}, \quad \widetilde{\mu}_{n}^{z}:=d_{2}^{-1} \mathbf{1}_{X_{\nu}} F^{*}\left(\widetilde{\mu}_{n-1}^{z}\right) .
$$

Les masses de $\widetilde{\mu}_{n}^{z}$ décroissent vers une constante $\tau_{\nu}(z)$. De la même manière que dans $[13, \S \S 3.4 .1-3.4 .3]$, on montre que

$$
\widetilde{\mathcal{E}}_{X_{\nu}}:=\bigcup_{n \geq 0} F^{n}\left(\mathcal{E}_{X_{\nu}}\right)
$$

contient l'ensemble $\left(\tau_{\nu}>0\right)$. La mesure $\mu_{n}^{z}$ étant égale à la moyenne des mesures $\mu_{m}^{a}$ avec $a \in F^{-n+m}(z)$ et $m \leq n$, si $\tau_{\nu}(z)=0$ la masse de $\mu_{\text {reg }}^{z}$ est, comme pour $z \notin X_{\nu}$, au moins égale à $1-\nu$. En particulier, $\mu_{\text {reg }}^{z}$ est de masse totale si $z$ n'appartient pas à $\bigcup_{\nu>0} \widetilde{\mathcal{E}}_{X_{\nu}}$. Dans ce cas, la mesure $\mu^{z}$ est égale à $\mu_{\text {reg }}^{z}$ et ne charge pas $\mathrm{PC}_{\infty}$. Ceci implique que $\mu_{n}^{z}\left(\mathrm{PC}_{\infty}\right)$ tend vers 0 . Or $\mathcal{E} \subset \mathrm{PC}_{\infty}$. On déduit qu'alors $\lim \mu_{n}^{z}=\mu$ et par conséquent $\mathcal{E}=\bigcup_{\nu>0} \widetilde{\mathcal{E}}_{\nu}$.

Montrons que $\mathcal{E}_{X_{\nu}}=\mathcal{E}_{X_{1}}$ pour tout $0<\nu<1$. Il est clair que $\mathcal{E}_{X_{\nu}} \supset \mathcal{E}_{X_{1}}$. Observons que d'après le théorème 3.9 , si $z \notin \mathcal{E}_{X_{1}}$ on a $\mu_{\text {reg }}^{z} \neq 0$. Si $z$ appartient à $\mathcal{E}_{X_{\nu}}$, les mesures $\mu_{n}^{z}$ sont portées par $X_{\nu}$. Donc $\mu_{\text {reg }}^{z}=0$ car $\mu$ ne charge pas les ensembles analytiques et $\mu_{n}^{z}$ est absolument continue par rapport à $\mu$. D'où on déduit que $z \in \mathcal{E}_{X_{1}}$.

On a montré que $\mathcal{E}=\widetilde{\mathcal{E}}_{X_{1}}$. Posons

$$
\mathcal{E}_{0}:=\bigcap_{n \geq 0} F^{-n}\left(\mathcal{E}_{X_{1}}\right)
$$

C'est un sous-ensemble algébrique de $\mathbb{C}^{k}$ vérifiant $F^{-1}\left(\mathcal{E}_{0}\right)=\mathcal{E}_{0}$. De plus, $\mathcal{E}_{0}$ est égal à l'intersection d'une famille finie d'ensembles algébriques $F^{-n}\left(\mathcal{E}_{X_{1}}\right)$. On a donc $\mathcal{E}=\widetilde{\mathcal{E}}_{X_{1}}=\bigcup_{n \geq 0} F^{n}\left(\mathcal{E}_{0}\right)$. Si $\mathcal{E}_{0}^{\prime}$ est un sous-ensemble algébrique vérifiant $F^{-1}\left(\mathcal{E}_{0}^{\prime}\right)=\mathcal{E}_{0}^{\prime}$, on montre comme on l'a fait pour $\mathcal{E}_{X_{\nu}}$ que $\mathcal{E}_{0}^{\prime} \subset \mathcal{E}_{X_{1}}$. La propriété $F^{-1}\left(\mathcal{E}_{0}^{\prime}\right)=\mathcal{E}_{0}^{\prime}$ implique que $\mathcal{E}_{0}^{\prime} \subset \mathcal{E}_{0}=\bigcap_{n \geq 0} F^{-n}\left(\mathcal{E}_{X_{1}}\right)$. D'où on déduit que $\mathcal{E}_{0}$ est le plus grand sous-ensemble algébrique propre de $\mathbb{C}^{k}$ invariant par $F^{-1}$.

Lorsque $F$ est une application polynomiale [13], on a $\mathcal{E}=\mathcal{E}_{0}$; l'existence de points périodiques répulsifs implique que la pseudo-métrique de Kobayashi de $\mathbb{C}^{k} \backslash \mathcal{E}_{0}$ est identiquement nulle; si $X$ est un sous-ensemble algébrique vérifiant $F^{-1}(X) \subset X$ alors $F^{-1}(X)=X$ et $F(X)=X$. Toutes ces propriétés sont fausses en général pour les correspondances comme on le voit dans les exemples suivants. 
Exemples 3.11. - Les correspondances suivantes sont polynomiales sur $\mathbb{C}$. Leurs exposants de Lojasiewicz sont strictement supérieurs à 1.

Considérons les polynômes d'une variable $f(z):=z^{3}$ et $g(z):=z^{2}-z$ et la correspondance $F:=f \circ g^{-1}$ sur $\mathbb{C}$ associée à la courbe $Y=\{(g(z), f(z)), z \in \mathbb{C}\}$ de $\mathbb{C}^{2}$. On vérifie que $\mathcal{E}_{0}=\{0\}$ et $\mathcal{E}=\bigcup_{n>0}\left(f \circ g^{-1}\right)^{n}(0)$. On vérifie aussi que 0 n'est pas isolé dans $\mathcal{E}$ et donc l'ensemble $\mathcal{E}$ est parfait.

Notons $\left(z_{1}, z_{2}\right)$ les coordonnées de $\mathbb{C}^{2}$. Soient $f, g$ deux polynômes d'une variable tels que $1<\operatorname{deg}(g)<\operatorname{deg}(f)$. Considérons la correspondance $F$ donnée par la courbe $Y:=\left\{\left[f\left(z_{1}\right)\right]^{2}+f\left(z_{2}\right) g\left(z_{2}\right)=0\right\}$. On a $F^{-1}(f=0)=(f=0)$ et donc $(f=0) \subset \mathcal{E}_{0}$. L'ouvert $\mathbb{C} \backslash \mathcal{E}_{0}$ est Kobayashi hyperbolique lorsque $f$ possède deux racines distinctes.

REMARQUE 3.12. - On peut étudier la restriction de la correspondance $F$ à $\mathcal{E}_{0}$, c'est-à-dire la correspondance sur $\mathcal{E}$ dont le graphe est égal à $Y \cap\left(\mathcal{E}_{0} \times \mathcal{E}_{0}\right)$, et construire sur $\mathcal{E}_{0}$ une mesure invariante par $F^{*}$ qui ne charge pas les sousensembles pluripolaires de $\mathcal{E}_{0}$. En faisant une récurrence descendante sur la dimension et sur le nombre de composantes du graphes, on montre que le cône des mesures positives $\nu$ qui vérifient $F^{*}(\nu)=d_{2} \nu$, est de dimension fini. Pour tout $z \in \mathbb{C}^{k}$, les valeurs adhérentes à la suite $\left(\mu_{n}^{z}\right)$ sont singulières par rapport à $\mu$ si et seulement si $z \in \mathcal{E}_{0}$ (voir aussi [12], [10]).

\section{Points périodiques répulsifs}

On appelle point fixe de $F$ tout point $z$ appartenant à l'ensemble $\pi_{2}(Y \cap$ Diag) où Diag est la diagonale de $\mathbb{C}^{k} \times \mathbb{C}^{k}$. Les points fixes isolés de $F$ sont comptés avec multiplicités. Les points périodiques de période $n$ sont les points fixes de $F^{n}$. On dira que $z_{0}$ est un point périodique régulier de période $n$ s'il existe une branche inverse régulière d'ordre $n$ d'un voisinage ouvert $K_{0}$ de $z_{0}$

$$
K_{-n},\left(\widehat{K}_{-n}, i_{n}\right), K_{-n+1},\left(\widehat{K}_{-n+1}, i_{n-1}\right), \ldots, K_{-1},\left(\widehat{K}_{-1}, i_{1}\right), K_{0}
$$

qui est accrochée à une branche inverse régulière $\mathcal{B}_{0}$ d'ordre $n$ de $z$ donnée par la suite

$$
z_{-n},\left(\widehat{z}_{-n}, i_{n}\right), z_{-n+1},\left(\widehat{z}_{-n+1}, i_{n-1}\right), \ldots, z_{-1},\left(\widehat{z}_{-1}, i_{1}\right), z_{0}
$$

avec $z_{-n}=z_{0}$. Si, de plus, les valeurs propres de la dérivée de $F_{\mathcal{B}_{0}}^{-n}$ en $z_{0}$ sont de module strictement plus petit que 1 , on dit que $z_{0}$ est périodique régulier répulsif. La proposition suivante donne le nombre de points périodiques (voir [10] pour le cas des correspondances sur les variétés kählériennes compactes).

Proposition 4.1. - Soit F une correspondance comme au théorème 2.1. Alors $F$ admet exactement $d_{2}^{n}$ points périodiques de période $n$ comptés avec multiplicités. Si $G$ est une autre correspondance polynomiale de degré topologique $\left(p_{1}, p_{2}\right)$ alors pour $n$ assez grand $G \circ F^{n}$ admet exactement $p_{2} d_{2}^{n}$ points fixes comptés avec multiplicités. 
Démonstration. - Puisque l'exposant de Lojasiewicz de $F$ est supérieur à 1, le choix de la boule $V$ entraîne que $(\operatorname{Diag} \cap Y) \subset(V \times V)$. De plus, on a $Y \cap(b V \times V)=\varnothing$. Pour tout $0 \leq t \leq 1$, posons

$$
\operatorname{Diag}(t):=\left\{(x, t x) \text { avec } x \in \mathbb{C}^{k}\right\} .
$$

C'est un sous-espace de $\mathbb{C}^{k} \times \mathbb{C}^{k}$ et on a Diag(1) = Diag. Comme $Y \cap(b V \times V)$ est vide, le nombre de points d'intersection de $Y \cap(V \times V)$ avec $\operatorname{Diag}(t)$ ne dépend pas de $t$. Quand $t=0$, ce nombre est exactement le nombre de préimages de 0 dans $V$ compté avec multiplicités; il est donc égal à $d_{2}$. Par conséquent, $F$ admet exactement $d_{2}$ points fixes. De même, on montre que $F^{n}$ admet exactement $d_{2}^{n}$ points fixes. Ce sont les points périodiques de période $n$ de $F$.

Pour $n$ assez grand, l'exposant de Lojasiewicz de $G \circ F^{n}$ est strictement plus grand que 1 . Par conséquent, $G \circ F^{n}$ admet exactement $p_{2} d_{2}^{n}$ points fixes.

Le théorème suivant généralise un résultat de Lyubich [26] qui a considéré le cas des fractions rationnelles de $\mathbb{P}^{1}$.

THÉORÈmE 4.2. - Soient F une correspondance comme au théorème 2.1 et $G$ une autre correspondance polynomiale de degré topologique $\left(p_{1}, p_{2}\right)$ et d'exposant de Lojasiewicz quelconque. Notons $\mathrm{PR}_{n}^{G}$ l'ensemble des points fixes réguliers répulsifs de $G \circ F^{n}$. Alors la suite de mesures

$$
\nu_{n}^{+}:=p_{2}^{-1} d_{2}^{-n} \sum_{z \in \mathrm{PR}_{n}^{G}} \delta_{z}
$$

tend faiblement vers la mesure d'équilibre $\mu$ de $F$.

En prenant $G(z)=z$, on obtient le corollaire suivant qui généralise un théorème de Lyubich [26], de Freire-Lopes-Mañé [20] et de Briend-Duval [3]. Ces auteurs ont considéré le cas des endomorphismes holomorphes de $\mathbb{P}^{k}$.

Corollaire 4.3. - Soit F une correspondance comme au théorème 2.1. Notons $\mathrm{PR}_{n}$ l'ensemble des points périodiques réguliers répulsifs de période $n$ de F. Alors la suite de mesures

$$
\nu_{n}:=d_{2}^{-n} \sum_{z \in \mathrm{PR}_{n}} \delta_{z}
$$

tend faiblement vers la mesure d'équilibre $\mu$ de $F$.

Nous allons adapter une méthode de Briend-Duval [3] qui traite le cas des endomorphismes holomorphes de $\mathbb{P}^{k}$. Il s'agit d'une application de la proposition 3.2 et du mélange sur une extension naturelle du système dynamique associé à $F$ (voir [8]). Cependant, les propriétés d'invariance et de mélange de la mesure d'équilibre dans notre cas sont plus faibles que dans le cas des endomorphismes. Plus précisément, la mesure $\mu$ n'est pas $F_{*}$-invariante, ce qui nous oblige à construire différemment la mesure associée à l'extension naturelle de $F$. Cette mesure ne sera pas invariante en général.

TOME $133-2005-\mathrm{N}^{\mathrm{O}} 3$ 
On a défini dans le lemme 3.4 un polynôme $P$ tel que tout point de $(P \neq 0)$ admet $d_{2}$ branches inverses régulières d'ordre 1 . Soit $Q$ un polynôme analogue associé à $G$. Notons $\Lambda$ l'opérateur de Perron-Frobenius associé à $F$ et $\Lambda_{G}$ celui associé à $G$. Notons $Z=\sum Z_{i}^{*}$ la $k$-chaîne holomorphe associée à $G$. Notons $X_{0}$ le complémentaire de $G\left(\mathcal{E}^{\prime}\right) \cup(Q=0)$ où $\mathcal{E}^{\prime}$ est l'orbite de $(P=0)$ par $F$. Du fait que $\mu$ ne charge pas les ensembles pluripolaires, $X_{0}$ est de $\mu$ mesure totale.

Dans la suite, nous allons changer un peu la terminologie « branche inverse régulière » pour l'adapter à la suite $\left(G \circ F^{n}\right)$. Soit $K_{1}$ un sous-ensemble connexe de $\mathbb{C}^{k}$. On appelle branche inverse régulière d'ordre $n$ de $K_{1}$ toute suite $\mathcal{B}$

$$
K_{-n},\left(\widehat{K}_{-n}, i_{n}\right), K_{-n+1},\left(\widehat{K}_{-n+1}, i_{n-1}\right), \ldots, K_{-1},\left(\widehat{K}_{-1}, i_{1}\right), K_{0},\left(\widehat{K}_{0}, i\right), K_{1}
$$

vérifiant les conditions suivantes

(i) Les ensembles $K_{-m} \subset \mathbb{C}^{k}$ sont connexes pour $m \geq-1, \widehat{K}_{-m} \subset Y_{i_{m}}^{*}$ pour $m \geq 1, \widehat{K}_{0} \subset Z_{i}^{*}$;

(ii) $\pi_{1}$ définit une bijection de $\widehat{K}_{-m}$ dans $K_{-m}$ et $\pi_{2}$ définit une bijection de $\widehat{K}_{-m}$ dans $K_{-m+1}$ pour $0 \leq m \leq n$.

Si $K_{1}$ n'est pas un ouvert (par exemple si $K_{1}$ est un point), on exige aussi que les applications ci-dessus définissent des bijections holomorphes entre un voisinage de $\widehat{K}_{-m}$ et ses images. Notons $\mathbf{F}_{\mathcal{B}}^{-m}$ l'application

$$
\left(\pi_{1 \mid \widehat{K}_{-m}}\right) \circ\left(\pi_{2 \mid \widehat{K}_{-m}}\right)^{-1} \circ \cdots \circ\left(\pi_{1 \mid \widehat{K}_{0}}\right) \circ\left(\pi_{2 \mid \widehat{K}_{0}}\right)^{-1}
$$

pour $1 \leq m \leq n$. C'est une application holomorphe bijective de $K_{1}$ dans $K_{-m}$. Les autres notations et la terminologie sont modifiées de la même manière.

Si $K_{1}$ est une boule et si $K_{-n}$ est strictement contenu dans $K_{1}$, alors l'application $\mathbf{F}_{\mathcal{B}}^{-n}$ est contractée pour la métrique de Kobayashi de $K_{1}$. Elle admet donc un point fixe attractif unique dans $K_{-n}$. C'est un point fixe répulsif de la correspondance $G \circ F^{n}$. Dans la suite, nous allons construire et compter les branches inverses régulières vérifiant cette propriété. Observons que tout point de $X_{0}$ admet exactement $p_{2} d_{2}^{n}$ branches inverses régulières d'ordre $n$.

Notons $X$ l'ensemble des branches inverses régulières d'ordre infini d'un point $x_{1} \in X_{0}$ définies par les suites infinies

$$
\ldots, x_{-n},\left(\widehat{x}_{-n}, i_{n}\right), x_{-n+1},\left(\widehat{x}_{-n+1}, i_{n-1}\right), \ldots, x_{-1},\left(\widehat{x}_{-1}, i_{1}\right), x_{0},\left(\widehat{x}_{0}, i\right), x_{1},
$$

$x$ désignera la suite infinie ci-dessus. La notation $X_{n}$ désignera l'ensemble des branches inverses régulières $x^{(n)}$ d'ordre $n$ données par les suites finies

$$
x_{-n},\left(\widehat{x}_{-n}, i_{n}\right), x_{-n+1},\left(\widehat{x}_{-n+1}, i_{n-1}\right), \ldots, x_{-1},\left(\widehat{x}_{-1}, i_{1}\right), x_{0},\left(\widehat{x}_{0}, i\right), x_{1} .
$$

Notons $\mathcal{A}_{0}$ la $\sigma$-algèbre des boréliens de $X_{0}$. Considérons la $\sigma$-algèbre $\mathcal{A}$ de $X$ engendrée par les ensembles

$$
A_{-m}(S):=\left\{x \in X, x_{-m} \in S\right\}
$$

BULletin DE LA SOCIÉtÉ MATHÉMATIQUE DE FRANCE 
où $S$ est un élément de $\mathcal{A}_{0}$ et $m \geq-1$. Définissons une mesure de probabilité $\widetilde{\mu}$ sur $X$. Soient $X_{-m}$ des éléments de $\mathcal{A}_{0}$. Posons

$$
\begin{gathered}
A\left(S_{1}, S_{0}, \ldots, S_{-m}\right):=A_{1}\left(S_{1}\right) \cap \cdots \cap A_{-m}\left(S_{-m}\right), \\
\tilde{\mu}\left(A\left(S_{1}, S_{0}, \ldots, S_{-m}\right)\right):=\left\langle\mu, \mathbf{1}_{S_{1}} \Lambda_{G}\left(\mathbf{1}_{S_{0}} \Lambda\left(\mathbf{1}_{S_{-1}}\left(\Lambda \mathbf{1}_{S_{-2}} \ldots\left(\Lambda \mathbf{1}_{S_{-m}}\right) \ldots\right)\right)\right)\right\rangle .
\end{gathered}
$$

Observons que la valeur de la fonction

$$
p_{2} d_{2}^{m} \mathbf{1}_{S_{1}} \Lambda_{G}\left(\mathbf{1}_{S_{0}} \Lambda\left(\mathbf{1}_{S_{-1}}\left(\Lambda \mathbf{1}_{S_{-2}} \ldots\left(\Lambda \mathbf{1}_{S_{-m}}\right) \ldots\right)\right)\right)
$$

en $x_{1}$ est égale au nombre de branches inverses d'ordre $m$

$$
x_{-m},\left(\widehat{x}_{-m}, i_{m}\right), x_{-m+1},\left(\widehat{x}_{-m+1}, i_{m-1}\right), \ldots, x_{-1},\left(\widehat{x}_{-1}, i_{1}\right), x_{0},\left(\widehat{x}_{0}, i\right), x_{1}
$$

de $x_{1}$ qui vérifient $x_{1} \in S_{1}, x_{0} \in S_{0}, \ldots, x_{-m} \in S_{-m}$.

On a la condition de compatibilité suivante

$$
\begin{aligned}
& \widetilde{\mu}\left(A \left(S_{1}, S_{0} \ldots,\right.\right.\left.\left.S_{-i+1}, S_{-i} \sqcup S_{-i}^{\prime}, S_{-i-1}, \ldots, S_{-m}\right)\right) \\
&=\widetilde{\mu}\left(A\left(S_{1}, S_{0}, \ldots, S_{-i+1}, S_{-i}, S_{-i-1}, \ldots, S_{-m}\right)\right) \\
& \quad+\widetilde{\mu}\left(A\left(S_{1}, S_{0}, \ldots, S_{-i+1}, S_{-i}^{\prime}, S_{-i-1}, \ldots, S_{-m}\right)\right)
\end{aligned}
$$

lorsque $S_{-i}, S_{-i}^{\prime}$ sont disjoints et $i \geq-1$. D'après le théorème de consistence de Kolmogoroff, $\widetilde{\mu}$ s'étend, de manière unique, en une mesure de probabilité sur $X$.

Notons $\Pi_{n}: X \rightarrow X_{n}$ la projection $\Pi_{n}(x):=x^{(n)}$ et $\tau_{n}: X_{n} \rightarrow X_{0}$ la projection $\tau_{n}\left(x^{(n)}\right):=x_{1}$. L'application $\tau_{n}$ est de degré $p_{2} d_{2}^{n}$ car on a supprimé tous les points donnant naissance à de mauvaises branches inverses. Pour $\varphi$ une fonction sur $X_{n}$ posons

$$
\left(\tau_{n}\right)_{*} \varphi(a):=\sum_{\tau_{n}(b)=a} \varphi(b)
$$

Lemme 4.4. - Soit A un élément de $\mathcal{A}$. Alors on a

$$
\widetilde{\mu}(A)=\lim _{n \rightarrow \infty} \int p_{2}^{-1} d_{2}^{-n}\left(\tau_{n}\right)_{*} \mathbf{1}_{\Pi_{n}(A)} \mathrm{d} \mu .
$$

Démonstration. - Il suffit de considérer le cas où $A=A\left(S_{1}, S_{0}, \ldots, S_{-m}\right)$. On a alors

$$
\widetilde{\mu}(A)=\int p_{2}^{-1} d_{2}^{-n}\left(\tau_{n}\right)_{*} \mathbf{1}_{\Pi_{n}(A)} \mathrm{d} \mu
$$

pour tout $n \geq m$. D'où le lemme.

Lemme 4.5. - Soit $S$ un élément de $\mathcal{A}_{0}$ et soit $A$ un élément de $\mathcal{A}$. Alors on a $\lim _{n \rightarrow \infty} \tilde{\mu}\left(A_{-n}(S) \cap A\right)=\mu(S) \widetilde{\mu}(A)$.

TOME $133-2005-\mathrm{N}^{\mathrm{O}} 3$ 
Démonstration. - Il suffit de considérer le cas où $A=A\left(S_{1}, S_{0}, \ldots, S_{-m}\right)$. Posons $c:=\mu(S)$ et $\varphi:=\mathbf{1}_{S}-c$. On a pour tout $n \geq m$

$$
\begin{gathered}
\left|\widetilde{\mu}\left(A_{-n}(S) \cap A\right)-c \widetilde{\mu}(A)\right|=\mid \int \mathbf{1}_{S_{1}} \Lambda_{G}\left(\mathbf{1}_{S_{0}} \Lambda\left(\mathbf{1}_{S_{-1}} \ldots \Lambda\left(\mathbf{1}_{S_{-m}} \Lambda^{n-m} \mathbf{1}_{S}\right) \ldots\right)\right) \mathrm{d} \mu \\
\quad-c \int \mathbf{1}_{S_{1}} \Lambda_{G}\left(\mathbf{1}_{S_{0}} \Lambda\left(\mathbf{1}_{S_{-1}} \ldots \Lambda\left(\mathbf{1}_{S_{-m}}\right) \ldots\right)\right) \mathrm{d} \mu \mid \\
=\left|\int \mathbf{1}_{S_{1}} \Lambda_{G}\left(\mathbf{1}_{S_{0}} \Lambda\left(\mathbf{1}_{S_{-1}} \ldots \Lambda\left(\mathbf{1}_{S_{-m}} \Lambda^{n-m} \varphi\right) \ldots\right)\right) \mathrm{d} \mu\right| \\
\leq \int \Lambda_{G} \Lambda^{m}\left|\Lambda^{n-m} \varphi\right| \mathrm{d} \mu .
\end{gathered}
$$

Notons $\mathbb{C}^{\perp}$ l'orthogonal de $\mathbb{C}$ dans $L^{2}(\mu)$. On a $\varphi \in \mathbb{C}^{\perp},\|\varphi\|_{\infty} \leq 1+|c|$ et donc $0 \leq \Lambda_{G} \Lambda^{m}\left|\Lambda^{n-m} \varphi\right| \leq 1+|c|$. D'après le théorème de convergence dominée, il suffit de montrer que pour tout $\psi \in \mathbb{C}^{\perp}, \Lambda^{n} \psi$ tend vers $0 \mu$-presque partout (extraire des sous-suites si nécessaire). Or, d'après le théorème 2.10, ceci est vrai pour $\psi$ lisse. Du fait que le sous-espace des fonctions lisses est dense dans $\mathbb{C}^{\perp}$, il suffit de montrer que $\|\Lambda\|_{\mathrm{L}^{2}(\mu)} \leq 1$. Grâce à l'inégalité de Cauchy-Schwarz et à l'invariance de $\mu$, on a

$$
\begin{aligned}
\int|\Lambda \psi(z)|^{2} \mathrm{~d} \mu(z) & =\int\left|\frac{1}{d_{2}} \sum_{w \in F^{-1}(z)} \psi(w)\right|^{2} \mathrm{~d} \mu(z) \\
& \leq \int \frac{1}{d_{2}}\left(\sum_{w \in F^{-1}(z)}|\psi(w)|^{2}\right) \mathrm{d} \mu(z)=\int \Lambda\left(|\psi|^{2}\right) \mathrm{d} \mu=\int|\psi|^{2} \mathrm{~d} \mu .
\end{aligned}
$$

Ceci termine la preuve du lemme.

Fin de la démonstration du théorème 4.2. - Posons, pour tout $\delta>0, E_{\delta}$ l'ensemble des $x \in X$ donnés par les suites

$$
\ldots, x_{-n},\left(\widehat{x}_{-n}, i_{n}\right), x_{-n+1},\left(\widehat{x}_{-n+1}, i_{n-1}\right), \ldots, x_{-1},\left(\widehat{x}_{-1}, i_{1}\right), x_{0},\left(\widehat{x}_{0}, i\right), x_{1}
$$

tels que la boule $\mathrm{B}\left(x_{1}, \delta\right)$ admette une branche inverse régulière d'ordre infini, de taille $\left(\ell^{-m / 4}\right)$ et accrochée à $x$. D'après la proposition 3.6, l'union $\bigcup_{\delta>0} E_{\delta}$ est de $\widetilde{\mu}$ mesure totale. Soit $\pi: X \longrightarrow X_{0}$ la projection $\pi(x):=x_{1}$. Posons

$$
\mu_{\delta}:=\pi_{*}\left(\mathbf{1}_{E_{\delta}} \tilde{\mu}\right)
$$

Cette mesure tend vers $\mu$ quand $\delta$ tend vers 0 . D'après la proposition 4.1, quand $n$ est assez grand, les mesures $\nu_{n}^{+}$sont de masse au plus 1 . Pour prouver le théorème 4.2 , on montre que toute valeur adhérente $\nu^{+}$à la suite $\left(\nu_{n}^{+}\right)$ vérifie $\mu_{\delta} \leq \nu^{+}$. Fixons $\delta, 0<\delta<1, \varepsilon, 0<\varepsilon<\delta$ et un point $z_{1} \in X_{0}$. Soit $r$ vérifiant $0<2 r<\delta-\varepsilon$. Il suffit de montrer que, pour tout $n$ assez grand,

$$
(1-\varepsilon) \mu_{\delta}\left(\overline{\mathrm{B}}\left(z_{1}, r\right)\right) \leq \nu_{n}^{+}\left(\mathrm{B}\left(z_{1}, r+\varepsilon\right)\right) .
$$

Posons $S:=\overline{\mathrm{B}}\left(z_{1}, r\right)$ et $A:=\pi^{-1}(S) \cap E_{\delta}$. D'après le lemme 4.5 , on a

$$
\lim _{n \rightarrow \infty} \tilde{\mu}\left(A_{-n}(S) \cap A\right)=\mu(S) \widetilde{\mu}(A)=\mu(S) \mu_{\delta}(S) .
$$

BULletin DE LA SOCIÉtÉ MATHÉMATIQUE DE FRANCE 
Soit $n$ assez grand tel que $\ell^{-n / 4}<\varepsilon$ et $\widetilde{\mu}\left(A_{-n}(S) \cap A\right)>(1-\varepsilon) \mu(S) \mu_{\delta}(S)$. Notons pour tout $z \in X_{0}, s(z)$ le nombre de branches inverses régulières $\mathcal{B}$ d'ordre $n$, de taille $\left(\ell^{-m / 4}\right)$ de $\mathrm{B}(z, \delta)$ telles que $\mathbf{F}_{\mathcal{B}}^{-n}(z) \in S$. On déduit de la dernière inégalité et du lemme 4.4 que pour $n$ assez grand

$$
\int p_{2}^{-1} d_{2}^{-n} s(z) \mathbf{1}_{S} \mathrm{~d} \mu \geq(1-\varepsilon) \mu(S) \mu_{\delta}(S) .
$$

Par conséquent, il existe un point $x_{1} \in S$ tel que $s\left(x_{1}\right) \geq(1-\varepsilon) p_{2} d_{2}^{n} \mu_{\delta}(S)$. Il existe donc au moins $(1-\varepsilon) p_{2} d_{2}^{n} \mu_{\delta}(S)$ branches inverses régulières $\mathcal{B}$ d'ordre $n$, de taille $\left(\ell^{-m / 4}\right)$ de $\mathrm{B}\left(x_{1}, \delta\right)$ telles que $z_{1}:=\mathbf{F}_{\mathcal{B}}^{-n}\left(x_{1}\right) \in S$. L'application $\mathbf{F}_{\mathcal{B}}^{-n}$ définit une application bijective de $\mathrm{B}\left(x_{1}, \delta\right)$ dans $W:=\mathbf{F}_{\mathcal{B}}^{-n} \mathrm{~B}\left(x_{1}, \delta\right)$. Or $W$ est un ensemble de diamètre plus petit que $\varepsilon$ et contient le point $z_{1} \in S$. On en déduit que $\bar{W}$ est contenu dans $\mathrm{B}\left(z_{1}, r+\varepsilon\right) \subset \mathrm{B}\left(x_{1}, \delta\right)$. Par conséquent, $\mathbf{F}_{\mathcal{B}}^{-n}$ admet un point fixe attractif unique dans $\mathrm{B}\left(z_{1}, r+\varepsilon\right)$. On en déduit que $\nu_{n}^{+}\left(\mathrm{B}\left(z_{1}, r+\varepsilon\right)\right) \geq(1-\varepsilon) \mu_{\delta}\left(\overline{\mathrm{B}}\left(z_{1}, r\right)\right)$. La démonstration du théorème 4.2 est achevée.

Remarque 4.6. - Dans le corollaire 4.3, on peut remplacer l'ensemble $\mathrm{PR}_{n}$ par l'ensemble $\operatorname{PR}_{n} \cap \operatorname{supp}(\mu)$. En effet, dans la preuve du théorème 4.2 pour le cas où $G(z)=z$, il suffit de considérer le point $x_{1}$ appartenant à $\operatorname{supp}(\mu)$. Or $\operatorname{supp}(\mu)$ est invariant par $F^{-1}$. Donc les points fixes obtenus appartiennent nécessairement à $\operatorname{supp}(\mu)$. On peut aussi remplacer $\mathrm{PR}_{n}$ par l'ensemble $\mathrm{PR}_{n}^{\prime}$ des point $x \in \mathrm{PR}_{n}$ dont la période minimale est égale à $n$. En effet, d'après la proposition 4.1 , on a $\#\left(\mathrm{PR}_{n} \backslash \mathrm{PR}_{n}^{\prime}\right)=\mathrm{o}\left(d_{2}^{n}\right)$.

Les corollaires suivants donnent des interprétations géométriques des résultats obtenus.

Corollaire 4.7. - Soient $F$ une correspondance comme au théorème 2.1 et $G$ une autre correspondance polynomiale de degré topologique $\left(p_{1}, p_{2}\right)$ associée à une $k$-chaîne holomorphe $Z$. Notons $Y_{n}$ la $k$-chaîne holomorphe associée à $F^{n}$ et $R_{n}$ l'intersection de $Y_{n}$ avec $Z$. Alors la suite de mesures

$$
p_{1}^{-1} d_{2}^{-n} \sum_{z \in R_{n}} \delta_{\pi_{1}(z)}
$$

tend faiblement vers la mesure d'équilibre $\mu$ de $F$.

Démonstration. - Soit $\bar{G}$ la correspondance adjointe de $G$. Chaque point fixe de $\bar{G} \circ F^{n}$ est associé à un couple de points $(x, y) \in Y_{n}$ et $(y, x) \in \bar{Z}$. On peut donc l'associer au point $(x, y)$ dans l'intersection de $Y_{n}$ avec $Z$. Il suffit maintenant d'appliquer le théorème 4.2 en remplaçant $G$ par $\bar{G}$.

Corollaire 4.8. - Soit F une correspondance comme au théorème 2.1. Soient $Y_{n}$ la k-chaîne holomorphe associée à $F^{n}$ et $\left[Y_{n}\right]$ le courant d'intégration sur $Y_{n}$. Alors le courant $d_{2}^{-n}\left[Y_{n}\right]$ tend faiblement vers le courant $\pi_{1}^{*}(\mu)$. 
Démonstration. - Pour toute matrice $A$ inversible de norme petite et de rang $k$, notons $\pi_{A}: \mathbb{C}^{k} \times \mathbb{C}^{k} \rightarrow \mathbb{C}^{k}$ l'application définie par

$$
\pi_{A}\left(z^{1}, z^{2}\right):=z^{2}-A z^{1} .
$$

Soit $\alpha:=\mathrm{d} z_{1} \wedge \mathrm{d} \bar{z}_{k} \wedge \cdots \wedge \mathrm{d} z_{k} \wedge \mathrm{d} \bar{z}_{k}$. Posons $\alpha_{A}:=\left(\pi_{A}\right)^{*} \alpha$. Les formes $\Phi \alpha_{A}$ avec $\Phi$ une fonction à support compact, engendrent l'espace des $(k, k)$-formes à support compact dans $\mathbb{C}^{k} \times \mathbb{C}^{k}$. Il suffit de montrer que

$$
\lim _{n \rightarrow \infty}\left\langle d_{2}^{-n}\left[Y_{n}\right]-\pi_{1}^{*}(\mu), \Phi \alpha_{A}\right\rangle=0 .
$$

Notons $E_{z}:=\pi_{A}^{-1}(z)$ pour tout $z \in \mathbb{C}^{k}$ et $\Phi_{z}$ la restriction de $\Phi$ à $E_{z}$. Notons également $\left[Y_{n}^{z}\right]$ et $\nu^{z}$ les mesures obtenues comme intersections de $\left[Y_{n}\right]$ et de $\pi_{1}^{*}(\mu)$ avec $E_{z}$. D'après le théorème de Fubini, on a

$$
\left\langle d_{2}^{-n}\left[Y_{n}\right]-\pi_{1}^{*}(\mu), \Phi \alpha_{A}\right\rangle=\int\left\langle d_{2}^{-n}\left[Y_{n}^{z}\right]-\nu^{z}, \Phi_{z}\right\rangle \mathrm{d} z_{1} \wedge \mathrm{d} \bar{z}_{1} \wedge \cdots \wedge \mathrm{d} z_{k} \wedge \mathrm{d} \bar{z}_{k} .
$$

D'après le corollaire $4.7,\left\langle d_{2}^{-n}\left[Y_{n}^{z}\right]-\nu^{z}, \Phi_{z}\right\rangle$ tend vers 0 pour tout $z$. Le théorème de convergence dominée implique que

$$
\lim _{n \rightarrow \infty}\left\langle d_{2}^{-n}\left[Y_{n}\right]-\pi_{1}^{*}(\mu), \Phi \alpha_{A}\right\rangle=0 .
$$

\section{Ensembles d'unicité pour les polynômes}

Soit $P$ un polynôme de degré $\operatorname{deg}(P) \geq 2$. Soient $K_{P}$ l'ensemble des points d'orbite bornée de $P$ et $J_{P}$ son bord topologique. L'ensemble $K_{P}$ s'appelle ensemble de Julia rempli de $P$ et $J_{P}$ est l'ensemble de Julia de $P$. D'après la théorie de Fatou, $J_{P}$ est le plus petit compact qui contient plus d'un point et qui vérifie $P^{-1}\left(J_{P}\right)=J_{P}$. L'ensemble $K_{P}$ est le plus grand compact vérifiant $P^{-1}\left(K_{P}\right)=K_{P}$. On dira qu'un compact $K$ est un ensemble d'allure Julia de $P$ si $K$ contient au moins deux points et si $P^{-1}(K)=K$. Il est clair qu'un tel ensemble $K$ n'est pas un ensemble d'unicité et vérifie $J_{P} \subset K \subset K_{P}$.

Considérons deux polynômes distincts non constants $f, g$ avec $\operatorname{deg}(f) \geq$ $\operatorname{deg}(g)$ et un compact $K$, vérifiant $\# K \geq 2$ et $f^{-1}(K)=g^{-1}(K)$. Dans [29] Ostrovskii, Pakovitch et Zaidenberg ont montré que si $\operatorname{deg}(f)=\operatorname{deg}(g)$, il existe une rotation $R$ préservant $K$ telle que $f=R \circ g$. Si la capacité logarithmique de $K$ est strictement positive, nous avons montré [9] qu'il existe un polynôme $P$ tel que $f=P \circ g$ et $P^{-1}(K)=K$ sauf pour les deux cas exceptionnels cidessous. Désignons par $z, Q, d, d^{\prime}$ et $a$ une coordonnée, un polynôme, deux entiers naturels et un nombre complexe convenables.

- Cas 1). - $K$ est une réunion de cercles de centre 0 et $f(z)=Q(z)^{d}$, $g=a Q(z)^{d^{\prime}}$.

- Cas 2). - $K$ est le segment $[-1,1]$ et $f= \pm \mathrm{T}_{d} \circ Q, g= \pm \mathrm{T}_{d^{\prime}} \circ Q$ où $\mathrm{T}_{m}$ est le polynôme de Tchebychev défini par la relation $\mathrm{T}_{m}(\cos z)=\cos (m z)$.

BULletin DE LA SOCIÉtÉ MATHÉMATIQUE DE FRANCE 
On voit que hors de ces deux cas si $\operatorname{deg}(f)>\operatorname{deg}(g), K$ est un ensemble d'allure Julia du polynôme $P$.

On déduit de ces résultats le théorème suivant.

ThÉORÈme 5.1. - Soient $f, g$ deux polynômes non constants avec $\operatorname{deg}(f) \geq$ $\operatorname{deg}(g)$. Soit $K$ un ensemble compact infini de $\mathbb{C}$ vérifiant $f^{-1}(K)=g^{-1}(K)$. Alors il existe un polynôme $P$ tel que $f=P \circ g$ et $P^{-1}(K)=K$ sauf si $(f, g, K)$ appartient à l'une des deux classes décrites ci-dessus.

Démonstration. - $\mathrm{Si} \operatorname{deg}(f)=\operatorname{deg}(g)$, ce théorème se réduit au résultat d'Ostrovskii-Pakovitch-Zaidenberg. Supposons que $\operatorname{deg}(f)>\operatorname{deg}(g)$. Posons $F:=f \circ g^{-1}$. C'est une correspondance polynomiale dont l'exposant de Lojasiewicz est égal à $\operatorname{deg}(f) \operatorname{deg}(g)^{-1}$. Elle vérifie l'hypothèse du corollaire 3.10. On a $F^{-1}(K)=K$ et $F(K)=K$. Comme $K$ est un ensemble infini, il n'est pas contenu dans l'ensemble $\mathcal{E}_{0}$. D'après le corollaire 3.10 et la remarque 3.12 , les relations $F^{-1}(K)=F(K)=K$ impliquent que $K$ contient le support de la mesure d'équilibre $\mu$ de $F$. La mesure $\mu$ étant PB, la capacité logarithmique de son support est strictement positive. Les résultats cités ci-dessus permettent de conclure.

Corollatre 5.2. - Un sous-ensemble compact infini de $\mathbb{C}$ est un ensemble d'unicité si et seulement s'il n'est pas un ensemble d'allure Julia et s'il n'est invariant par aucune rotation.

\section{BIBLIOGRAPHIE}

[1] Alexander (H.) - Projective capacity, Ann. Math. Studies, vol. 100, Princeton University Press, 1981, pp. 3-27.

[2] Bedford (E.) \& SMillie (J.) - External rays in dynamics of polynomial automorphisms of $\mathbb{C}^{2}$, vol.222, American Mathematical Society, Providence, RI, 1999, pp. 41-79.

[3] Briend (J.Y.) \& Duval (J.) - Exposants de Liapounoff et distribution des points périodiques d'un endomorphisme de $\mathbb{C P}^{k}$, Acta Math., t. 182 (1999), pp. 143-157.

[4] _ Deux caractérisations de la mesure d'équilibre d'un endomorphisme de $\mathbb{P}^{k}(\mathbb{C})$, Publ. Math. Inst. Hautes Études Sci., t. 93 (2001), pp. $145-159$.

[5] Brolin (H.) - Invariant sets under iteration of rational functions, Ark. Mat., t. 6 (1965), pp. 103-144.

[6] Clozel (L.) \& Ullmo (E.) - Correspondances modulaires et mesures invariantes, J. reine angew. Math., t. 558 (2003), pp. 47-83.

TOME $133-2005-\mathrm{N}^{\mathrm{O}} 3$ 
[7] _ Équidistribution des points de Hecke, in Contributions to automorphic forms, geometry, and number theory, Johns Hopkins Univ. Press, Baltimore, MD, 2004, pp. 193-254.

[8] Cornfeld (I.P.), Fomin (S.V.) \& Sinai (Y.G.) - Ergodic theory, Grundlehren Math. Wiss., vol. 245, Springer Verlag, New York-Berlin, 1962.

[9] Dinh (T.-C.) - Ensembles d'unicité pour les polynômes, Ergodic Theory Dynamical Systems, t. 22 (2002), pp. 171-186.

[10] Suites d'applications méromorphes multivaluées et courants laminaires, J. Geom. Anal. (à paraître).

[11] Dinh (T.-C.) \& SibOny (N.) - Sur les endomorphismes holomorphes permutables de $\mathbb{P}^{k}$, Math. Ann., t. 324 (2002), pp. 33-70.

[12] _ Distribution des valeurs de transformations méromorphes et applications, prépublication, arXiv : math.DS/0306095, 2003.

[13] _ Dynamique des applications d'allure polynomiale, J. Math. Pures Appl., t. 82 (2003), pp. 367-423.

[14] _ Dynamique des applications polynomiales semi-régulières, Ark. Mat., t. 42 (2004), pp. 61-85.

[15] _ Green currents for holomorphic automorphisms on compact Kähler manifolds, J. Amer. Math. Soc., t. 18 (2005), pp. 291-312.

[16] EREmenko (A.E.) - On some functional equations connected with iteration of rational function, Leningrad Math. J., t. 1 (1990), pp. 905-919.

[17] FAtou (P.) - Sur l'itération analytique et les substitutions permutables, J. Math., t. 2 (1923), pp. 343.

[18] Fornæss (J.E.) - Dynamics in several complex variables, CBMS, vol. 81, Amer. Math. Soc., Providence, RI, 1996.

[19] Fornæss (J.E.) \& Sibony (N.) - Complex dynamics in higher dimension, in Complex potential theory (Montréal, PQ, 1993), Nato ASI Series Math. and Phys. Sci., vol. C439, Kluwer, 1994, pp. 131-186.

[20] Freire (A.), Lopes (A.) \& MAÑ́ (R.) - An invariant measure for rational maps, Bol. Soc. Brasil. Mat., t. 14 (1983), pp. 45-62.

[21] Gross (F.) \& YANG (C.C.) - On preimage and range sets of meromorphic functions, Proc. Japan Acad. Ser. A Math. Sci., t. 58 (1982), pp. 17 20.

[22] Guedu (V.) - Ergodic properties of rational mappings with large topological degree, prépublication, 2003.

[23] HöRmander (L.) - The analysis of linear partial differential operators I, Springer-Verlag, 1983.

[24] Julia (G.) - Mémoire sur la permutabilité des fractions rationnelles, Ann. Sci. École Norm. Sup., t. 39 (1922), pp. 131-215.

[25] KlimeK (M.K.) - Pluripotential theory, Oxford Univ. Press, 1995. 
[26] Lyubich (M.Ju.) - Entropy properties of rational endomorphisms of the Riemann sphere, Ergodic Theory Dynamical Systems, t. 3 (1983), pp. 351385.

[27] MÉo (M.) - Image inverse d'un courant positif fermé par une application surjective, C. R. Acad. Sci. Paris Sér. I Math., t. 322 (1996), pp. 11411144.

[28] Nevanlinna (R.) - Einige Eideutigkeitss atze in der Theorie der meromorphen Funktionen, Acta Math., t. 48 (1926), pp. 367-391.

[29] Ostrovskit (I.V.), Pakovitch (F.B.) \& Zaidenberg (M.G.) - A remark on complex polynomial of least deviation, Internat. Math. Res. Notices, t. 14 (1996), pp. 699-703.

[30] Ploski (A.) - On the growth of proper polynomial mappings, Ann. Polon. Math., t. XLV (1985), pp. 297-309.

[31] Ritt (J.F.) - Permutable rational functions, Trans. Amer. Math. Soc., t. 25 (1923), pp. 399-448.

[32] Russakovskit (A.) \& Shiffman (B.) - Value distribution for sequences of rational mappings and complex dynamics, Indiana Univ. Math. J., t. 46 (1997), pp. 897-932.

[33] ShifFmAN (B.) - Uniqueness of entire and meromorphic functions sharing finite sets, Complex Variables Theory Appl., t. 43 (2001), pp. 433-449.

[34] Sibony (N.) - Dynamique des applications rationnelles de $\mathbb{P}^{k}$, in Dynamique et géométrie complexes, Panoramas \& Synthèses, vol. 8, Société Mathématique de France, Paris, 1999, pp. 97-185.

[35] Sibony (N.) \& Wong (P.M.) - Some results on global analytic sets, in Séminaire Lelong-Skoda, Lect. Notes in Math., vol. 822, Springer, 1980, pp. 221-237.

[36] Voisin (C.) - Intrinsic pseudovolume forms and $K$-correspondences, in The Fano Conference, Univ. Torino, Turin, 2004, pp. 761-792. 